ARTICLE

\title{
A stem cell reporter based platform to identify and target drug resistant stem cells in myeloid leukemia
}

Kyle Spinler 1,2, Jeevisha Bajaj (10 1,2, Takahiro Ito (1) 1,2, Bryan Zimdahl1,2,3, Michael Hamilton 1,2, Armin Ahmadi ${ }^{1,2}$, Claire S. Koechlein ${ }^{1,2}$, Nikki Lytle1,2, Hyog Young Kwon (10) 1,2, Ferdous Anower-E-Khuda4, Hao Sun (D) ${ }^{5}$, Allen Blevins ${ }^{1,2}$, Joi Weeks ${ }^{1,2}$, Marcie Kritzik ${ }^{1,2}$, Jan Karlseder (D) ${ }^{6}$, Mark H. Ginsberg (iD ${ }^{5}$, Pyong Woo Park ${ }^{7}$, Jeffrey D. Esko (i) ${ }^{4}$ \& Tannishtha Reya (1) $1,2,5,8 \otimes$

Intratumoral heterogeneity is a common feature of many myeloid leukemias and a significant reason for treatment failure and relapse. Thus, identifying the cells responsible for residual disease and leukemia re-growth is critical to better understanding how they are regulated. Here, we show that a knock-in reporter mouse for the stem cell gene Musashi 2 (Msi2) allows identification of leukemia stem cells in aggressive myeloid malignancies, and provides a strategy for defining their core dependencies. Specifically, we carry out a high throughput screen using Msi2-reporter blast crisis chronic myeloid leukemia (bcCML) and identify several adhesion molecules that are preferentially expressed in therapy resistant bcCML cells and play a key role in bcCML. In particular, we focus on syndecan-1, whose deletion triggers defects in bcCML growth and propagation and markedly improves survival of transplanted mice. Further, live imaging reveals that the spatiotemporal dynamics of leukemia cells are critically dependent on syndecan signaling, as loss of this signal impairs their localization, migration and dissemination to distant sites. Finally, at a molecular level, syndecan loss directly impairs integrin $\beta_{7}$ function, suggesting that syndecan exerts its influence, at least in part, by coordinating integrin activity in bcCML. These data present a platform for delineating the biological underpinnings of leukemia stem cell function, and highlight the Sdc1-Itg $\beta 7$ signaling axis as a key regulatory control point for bcCML growth and dissemination.

\footnotetext{
${ }^{1}$ Department of Pharmacology, University of California San Diego School of Medicine, La Jolla, CA, USA. ${ }^{2}$ Sanford Consortium for Regenerative Medicine, La Jolla, CA, USA. ${ }^{3}$ Department of Pharmacology and Cancer Biology, Duke University Medical Center, Durham, NC, USA. ${ }^{4}$ Department of Cellular and Molecular Medicine, University of California San Diego School of Medicine, La Jolla, CA, USA. ${ }^{5}$ Department of Medicine, University of California San Diego, La Jolla, CA, USA. ${ }^{6}$ Salk Institute, La Jolla, CA, USA. ${ }^{7}$ Department of Medicine, Boston Children's Hospital, Harvard Medical School, Boston, MA, USA.

${ }^{8}$ Moores Cancer Center, University of California San Diego School of Medicine, La Jolla, CA, USA. ${ }^{凶}$ email: treya@health.ucsd.edu
} 
O ver the past several decades, it has become increasingly clear that many cancers are heterogeneous and contain a distinct population of tumor-propagating cells that can also be highly resistant to anticancer therapies ${ }^{1-5}$. Existence of such a therapy-resistant population can explain why many cancers re-emerge after treatment. A key example of this is in chronic myelogenous leukemia (CML), where CML stem cells remain therapy resistant and cannot be eliminated by imatinib (Gleevec), thus resulting in a continued dependence on the drug for a patient's lifetime. In addition, with the accrual of additional mutations, CML progresses to the aggressive blast crisis phase, which is highly undifferentiated and largely unresponsive to Gleevec $^{6-10}$. Similarly, stem cells in de novo acute myeloid leukemia have been shown to express elevated levels of multidrug resistance genes and exhibit reduced sensitivity to daunorubicin, a standard of care chemotherapeutic in AML ${ }^{11-13}$.

To date, therapy-resistant stem cells in leukemias have predominantly been identified through combinations of surface markers ${ }^{1,14-17}$. While these approaches have been important for prospective isolation of cells and assessment of their role in cancer initiation and propagation, they are limited in their ability to identify and track stem cells in vivo, and cannot be effectively integrated into strategies to screen for dependencies. Thus, the development of efficient ways to identify leukemia stem cells (LSCs) is critically needed to enable real-time detection of therapy-resistant cells, and improve the delineation of their biological underpinnings.

Here we show that a knock-in reporter for the stem cell gene Musashi 2 (Msi2) ${ }^{18-20}$ can serve as a platform to define functional heterogeneity in hematological malignancies, and effectively identify LSCs in both blast crisis chronic myeloid leukemia (bcCML) and de novo AML. By screening for dependencies using this approach, we find several adhesion molecules enriched in therapy-resistant bcCML cells. Among these, syndecan and syndecan-dependent integrin signaling are not only essential for bcCML propagation and lethality but they also control systemic dissemination to distant sites. These data show that Msi reporters represent a key strategy for identifying regulators of LSCs, and highlight adhesive signals as critical players in LSC growth and dissemination.

\section{Results}

Functional heterogeneity defined by Msi2 in hematologic malignancies. To test if Msi2-reporter activity can effectively identify LSCs and therapy resistance in myeloid leukemia, we used a BCR-ABL/NUP98-HOXA9-driven model of bcCML ${ }^{21}$. A majority (77\%) of bcCML cells derived from Msi2-reporter KLS cells were GFP-positive (Fig. 1a, b), with reporter activity marking the most immature population within bcCML ( Lin $^{-}$) (Fig. 1c, d). $\mathrm{GFP}^{+}$cells were also more leukemogenic, as they formed 14.5fold more colonies than $\mathrm{GFP}^{-}$cells (Fig. 1e), consistent with prior findings on $\mathrm{Lin}^{-}$bcCML cells ${ }^{22}$. Interestingly, Msi2-reporter expression further fractionated $\mathrm{Lin}^{-}$cells into $\mathrm{GFP}^{+}$and $\mathrm{GFP}^{-}$ populations (Fig. 1f) with $\mathrm{Lin}^{-} \mathrm{GFP}^{+}$cells exhibiting significantly greater colony-forming ability (Fig. 1g), indicating that Msi2reporter expression marks the more tumorigenic cells within the immature fraction of bcCML. To define whether $\mathrm{GFP}^{+}$leukemia cells are enriched for stem cell activity in vivo, $\mathrm{GFP}^{+}$and $\mathrm{GFP}^{-}$ bcCML cells were transplanted and leukemia development monitored. While none of the mice transplanted with $\mathrm{GFP}^{-}$cells developed leukemia, all of the mice transplanted with $\mathrm{GFP}^{+}$cells succumbed to bcCML (Fig. 1h, i), suggesting that the majority of LSC activity in vivo resides within the Msi2-expressing $\mathrm{GFP}^{+}$ fraction of the population. We confirmed that the bcCML LSC population (as characterized by Neering et al. ${ }^{22}$ ) lies exclusively within the Msi2-GFP ${ }^{+}$population (Supplementary Fig 1a). While only $\sim 0.1 \%$ of $\mathrm{Msi} 2-\mathrm{GFP}^{+}$cells are LSCs, $100 \%$ of LSCs were exclusively Msi2-GFP ${ }^{+}$. To determine if the functional demonstration of LSC activity aligned with a molecular LSC signature, we tested whether genes in the previously reported molecular signature of AML LSCs ${ }^{23}$ were also preferentially enriched in Msi2-reporter ${ }^{+}$bcCML cells. Using RNA Seq analysis of bcCML $\mathrm{Lin}^{-}$cells we assessed expression levels of the mouse homologues of the genes comprising the human leukemic stem cell gene signature (Supplementary Fig $1 \mathrm{~b}$ ). Of these, we selected a subset and directly compared expression in Msi2-reporter ${ }^{+}$bcCML and Msi2-reporter ${ }^{-}$bcCML cells by PCR, and found that these genes (Map3k7, Ppig, Rbpms, Tgif2, and Vgll4) were preferentially higher in Msi2-reporter ${ }^{+}$bcCML cells relative to the Msi2reporter $^{-}$cells (Supplementary Fig. 1c). These data provide a molecular correlate for our finding that Msi2-reporter ${ }^{+}$cells are functionally enriched in LSC activity.

Interestingly Msi2-reporter activity may mark stem cells in some other hematologic malignancies as well: in an MLL-AF9/

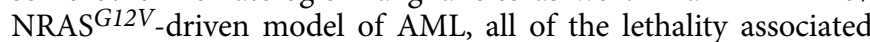
with the disease was harbored within the $\mathrm{GFP}^{+}$leukemia cells (Fig. 1j, k) ${ }^{24}$. The Msi2 reporter also marked LSCs in chronic myeloid leukemia (Fig. 11) and in an NPM1-driven model of myeloproliferative disease (Fig. $1 \mathrm{~m}$ ). These data collectively suggest that the Msi2 reporter could serve as a more general strategy to identify stem cells in myeloid malignancies.

Therapy-resistant population defined by Msi2-GFP reporter activity. Because imatinib is significantly less effective as CML progresses to bcCML ${ }^{25,26}$, we tested if Msi2-reporter activity also identifies the therapy-resistant cells within bcCML. We specifically analyzed cell survival of $\mathrm{GFP}^{+}$and $\mathrm{GFP}^{-}$cells within $\mathrm{Lin}^{-}$cells to account for any differences that may be solely due to differentiation status. While only $14 \%$ of $\mathrm{Lin}^{-} \mathrm{GFP}^{-}$cells were viable after imatinib treatment (Fig. $2 \mathrm{a}, \mathrm{b}$ ), $\mathrm{GFP}^{+}$cells were strikingly more resistant with $86 \%$ of $\mathrm{Lin}^{-} \mathrm{GFP}^{+}$cells remaining viable (Fig. 2a, b). We analyzed this therapy response heterogeneity within the Msi2-reporter ${ }^{+}$population by analyzing the LSC compartment after exposure to imatinib. We sorted Msi2-GFP ${ }^{+}$ and Msi2-GFP ${ }^{-}$bcCML cells, incubated with imatinib overnight, and analyzed the bcCML LSC population ( $\mathrm{Lin}^{-} \mathrm{CD} 150^{-}$Flt3+ $\mathrm{Sca}^{-22}$ ) by flow cytometry. In support of our finding that the LSC population is exclusive to the Msi2-GFP+ fraction, we found that after incubation with imatinib, the LSC content within the $\mathrm{Msi}-\mathrm{GFP}^{+}$fraction was enriched (Supplementary Fig. 1d). This suggests that the LSCs represent the most therapy-resistant cells within the $\mathrm{Msi2}-\mathrm{GFP}^{+}$population and endows this population with its chemotherapy resistance and disease propagation capacity. Msi2-reporter activity also marked cells resistant to DNA damaging agents. $96 \%$ of the $\mathrm{Lin}^{-} \mathrm{GFP}^{+}$cells survived radiation exposure compared to $47 \%$ of $\mathrm{Lin}^{-} \mathrm{GFP}^{-}$cells (Fig. 2c). Further, while $\mathrm{GFP}^{-}$cells showed increased sensitivity to increasing doses of radiation (0-10 Gy) (Fig. $2 \mathrm{~d}$ ), $\mathrm{GFP}^{+}$cells remained highly resistant, exhibiting 93\% viability even at the highest radiation dose used (10 Gy, Fig. 2d). Collectively, these data show that the Msi2-reporter model can be an effective tool to identify therapyresistant cells in myeloid leukemia.

The fact that Msi2 ${ }^{+}$leukemia cells had a greater capacity to propagate disease and were preferentially able to evade therapy suggested that the reporter might serve as a particularly important tool to identify regulators of these cells. Based on this, we undertook an unbiased high throughput screen to define key dependencies of $\mathrm{Msi}^{+}$bcCML cells. The screen was focused specifically on cell surface proteins as these provide the main mechanism through which leukemia cells would interact with 
a

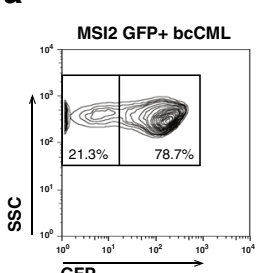

GFP

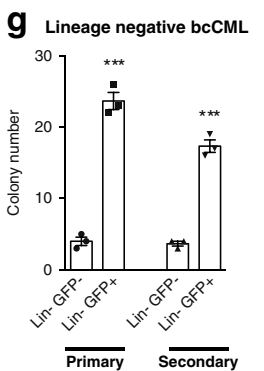

b

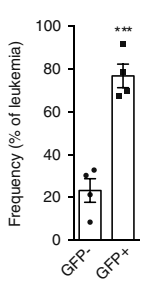

c

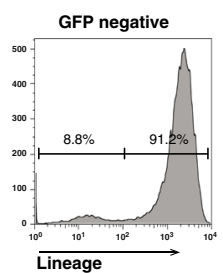

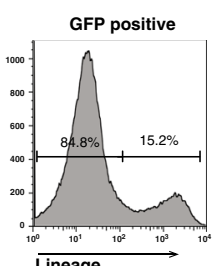

h

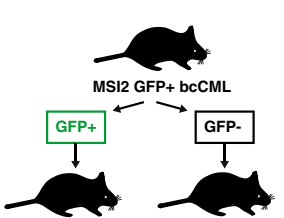

i

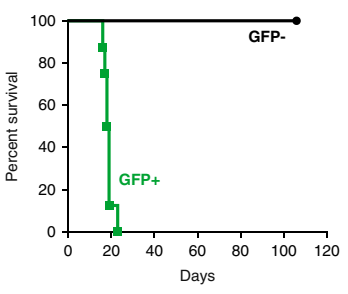

d

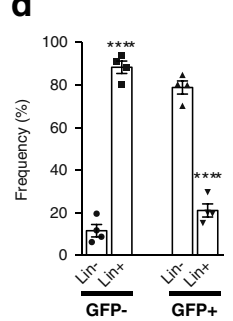

j

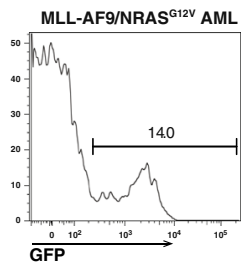

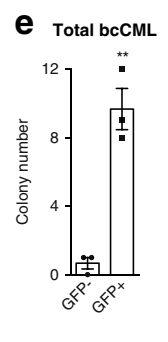

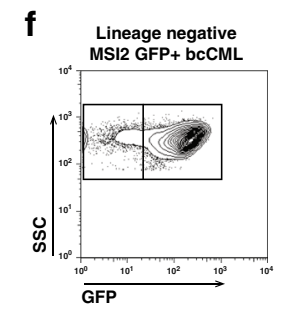

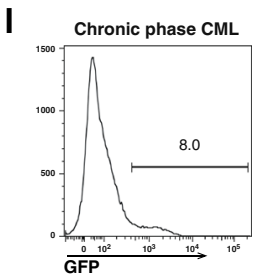
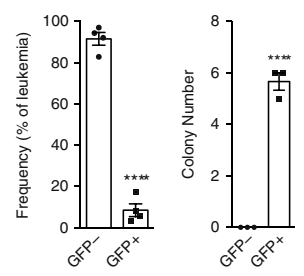

m
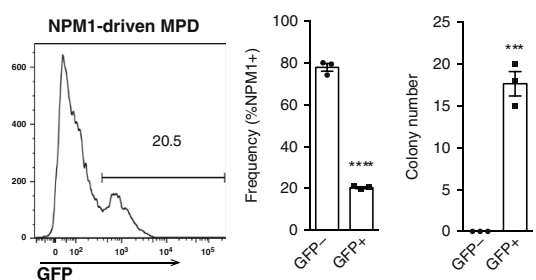

k
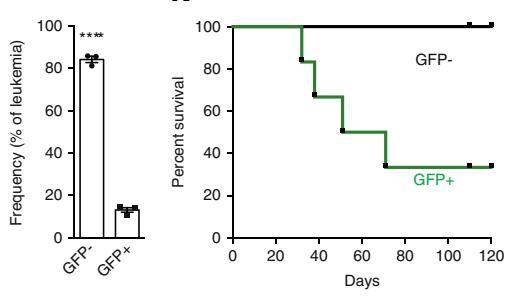

Fig. 1 Msi2 marks leukemia stem cells in a mouse model of bcCML. a Representative FACS plot shows GFP expression in Msi2-reporter bcCML. b Average frequency of $\mathrm{GFP}^{-}$negative (GFP-) and GFP-positive (GFP+) leukemic spleen cells ${ }^{\star \star \star} P=0.0005$ ( $n=4$ mice). c Representative histograms show lineage expression in GFP- and GFP + leukemic spleen cells. $\mathbf{d}$ The average frequency of lineage-negative (Lin ${ }^{-}$) and lineage-positive (Lin ${ }^{+}$) cells within either the GFP- or GFP+ fraction ${ }^{\star \star \star \star} P<0.0001$ ( $n=4$ mice per group). e Number of colonies generated from GFP ${ }^{-}$and GFP + bcCML cells. ${ }^{\star \star} P=$ 0.002 ( $n=3$ technical replicates). $\mathbf{f}$ Representative FACS plot shows GFP expression within the lineage-negative (Lin ${ }^{-}$) fraction of the spleen from a leukemic mouse. $\mathbf{g}$ Number of colonies generated from Lin- GFP- and Lin - GFP + bcCML cells after primary and secondary plating. ${ }^{\star \star \star} P=0.0001(n=3$ technical replicates each). $\mathbf{h}$ Schematic illustrates an experimental approach to test the ability of established GFP + and GFP- bcCML cells to drive disease development in secondary recipient mice. GFP ${ }^{+}$or $\mathrm{GFP}^{-}$cells from established bcCML were transplanted into recipients and $\mathbf{i}$ survival was monitored $(n=$ 8 for $\mathrm{GFP}^{+}$and $n=10$ for $\mathrm{GFP}^{-}$). $\mathbf{j}$ The representative histogram shows GFP expression in leukemic spleen cells from a mouse with MLL-AF9/NRASG12V driven AML (left panel); frequency of GFP- and GFP+ leukemic spleen cells from mice with MLL-AF9/NRASG12V-driven AML ${ }^{\star \star \star \star} P<0.0001$ (right panel; $n=3$ mice per group). $\mathbf{k}$ Survival of mice transplanted with GFP- ${ }^{-}$and GFP ${ }^{+}$MLL-AF9/NRASG12V -driven AML $(n=6$ mice per cohort). I The representative histogram shows GFP expression in leukemic spleen cells from a mouse with BCR-ABL-driven CML (left panel); frequency of GFP- and GFP+ leukemic spleen cells from mice with BCR-ABL-driven $C M L{ }^{\star \star \star \star} P<0.0001$ (middle panel, $n=4$ mice per group); colony formation of Msi2-reporter ${ }^{+}$and $M$ Msi2- $^{2}$ reporter- $\mathrm{CML}^{\star \star \star \star} \mathrm{P}<0.0001$ (right panel; $n=3$ mice). $\mathbf{m}$ The representative histogram shows GFP expression in leukemic spleen cells from a mouse with NPM1-driven MPD (left panel); frequency of GFP- and GFP+ leukemic spleen cells from mice with NPM1-driven MPD ${ }^{\star \star \star \star} P<0.0001$ (middle panel, $n=3$ mice per group); colony formation of Msi2-reporter ${ }^{+}$and Msi2-reporter- MPD ${ }^{\star \star \star} P=0.0003$ (right panel; $n=3$ mice per group). Two-tailed unpaired Student's $t$-tests were used to determine statistical significance. Source data are provided as a Source Data File.

cells of the microenvironment. In part this decision was based on live imaging showing that leukemia cells are in fact highly interactive with local niches, suggesting that these associations could provide critical cues for their survival and continued propagation (Fig. 2e and Supplementary Movie 1).

High throughput screen to identify dependencies of $\mathrm{Msi2}^{+}$ bcCML cells. The high throughput screen employed commercially available antibody plates to test a large set of monoclonal antibodies (mAbs) against 176 surface proteins, including 22 IgG isotype control antibodies (Fig. 3a). bcCML cells were incubated in the presence of the antibodies, and subsequently analyzed by flow cytometry to determine expression on $\mathrm{GFP}^{+}$and $\mathrm{GFP}^{-}$cells. Of the $176 \mathrm{mAbs}$ tested, 53 were found to be absent on bcCML cells ( $<5 \%$ of cells positive), and 62 were expressed highly on both $\mathrm{GFP}^{+}$and $\mathrm{GFP}^{-}$cells ( $>10 \%$ of cells positive). We focused specifically on proteins expressed preferentially on $\mathrm{GFP}^{+}$cells, as they could potentially be important for driving differential programs in $\mathrm{Msi} 2^{+}$cells. A total of 41 proteins displayed expression greater than twofold higher on $\mathrm{GFP}^{+}$cells compared to $\mathrm{GFP}^{-}$ cells. These included proteins that have previously been implicated in CML and bcCML, such as CD $34^{22}, \mathrm{CD} 41^{27}, \mathrm{CD} 119^{28}$, and $\mathrm{CD} 274^{29}$, serving as an independent validation of this approach. Most importantly, this approach identified multiple surface molecules whose role in myeloid leukemia remains unknown; these included integrin $\beta_{7}$, siglec-F (CD170), CD72, LPAM-1 (integrin $\alpha_{4} \beta_{7}$ ) and syndecan-1 (Sdc1, CD138) (Fig. 3a-c).

Given the potential for discovering new programs important for myeloid leukemia, we selected the subset for which blocking antibodies were available and could thus be considered for targeting. Thus we focused on $\operatorname{Sdc1}$, Itg $\beta 7$, and $\alpha_{4} \beta_{7}$, whose expression on bcCML cells was confirmed by subsequent flow cytometry analysis and found to be present at the ratios detected 
a

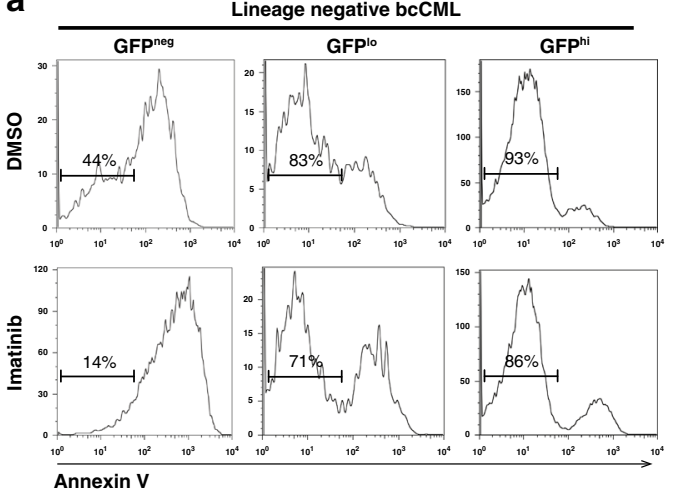

C

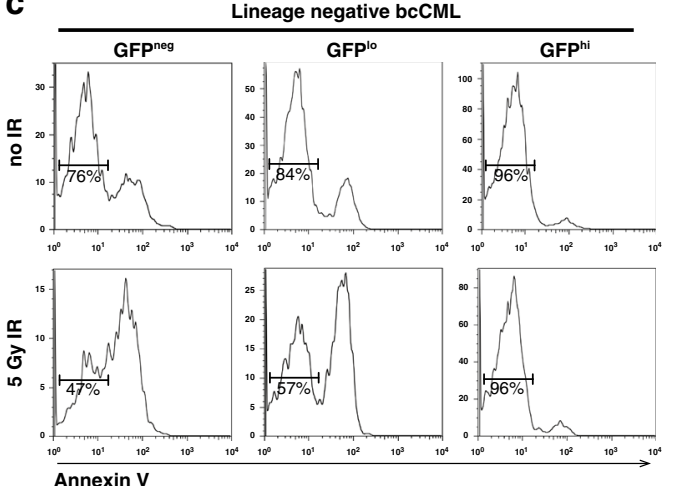

b

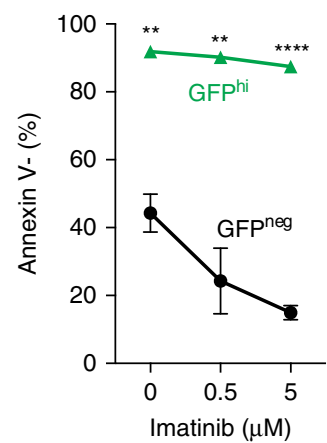

e

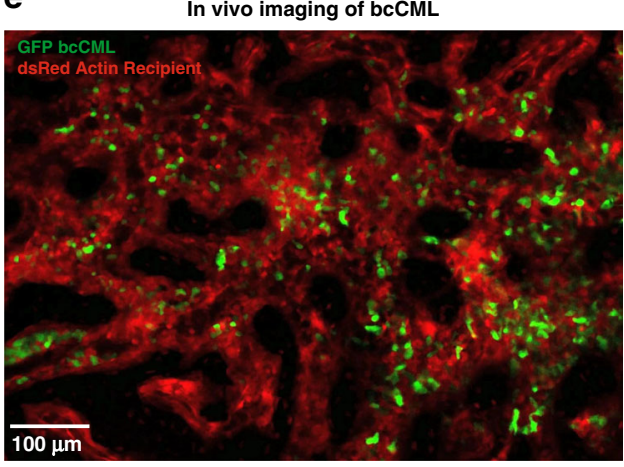

d

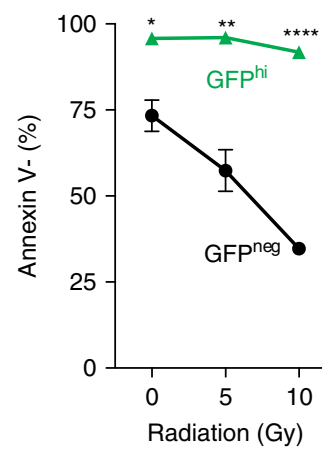

Fig. 2 Msi2 marks bcCML cells that are highly therapy resistant. a, b Msi2-expressing (GFPhi) cells are highly resistant to imatinib-induced cell death. a Representative histograms show frequency of live (Annexin V-) GFPneg, GFPlo and GFPhi established lineage-negative bcCML cells after $7 \mathrm{~h}$ of imatinib $(5 \mu \mathrm{M})$ or DMSO control treatment. $\mathbf{b}$ The average frequency of live (Annexin V-) GFPhi and GFPneg cells after $7 \mathrm{~h}$ of imatinib (500 $\mathrm{nM}$ or $5 \mu \mathrm{M}$ ) or DMSO control treatment. ${ }^{\star \star} P=0.001$ for $0 \mu \mathrm{M}$, ${ }^{\star \star} P=0.002$ for $0.5 \mu \mathrm{M}$, ${ }^{\star \star \star \star} P<0.0001$ for $5 \mu \mathrm{M}$ ( $n=4$ for each treatment condition and cell type). $\mathbf{c}$, $\mathbf{d} \mathrm{Msi} 2$ expressing (GFPhi) cells are highly resistant to radiation-induced cell death. c Representative histograms show frequency of live (Annexin $V$-) GFPneg, GFPlo and GFPhi established lineage-negative bcCML cells $7 \mathrm{~h}$ following radiation ( $5 \mathrm{~Gy}$ ). d The average frequency of live (Annexin $\mathrm{V}$-) GFPhi and GFPneg cells $7 \mathrm{~h}$ following radiation $(0,5$, or $10 \mathrm{~Gy}) .{ }^{\star} P=0.01,{ }^{\star \star} P=0.003,{ }^{\star \star \star \star} P<0.0001$ ( $n=3$ for each treatment condition and cell type). e Representative live animal imaging of the calvarium after the establishment of GFP+ bcCML; green, donor mouse: actin GFP lin- bcCML, red, recipient mouse: actin dsRed (See also Supplementary Movie 1). Two-tailed unpaired Student's $t$-tests were used to determine statistical significance. Source data are provided as a Source Data File.

in the screen (Fig. 3d). We also validated the results of our screen using antibodies against a number of proteins that were downregulated (Supplementary Fig. 1e) or unchanged (Supplementary Fig. 1f). To assess their relative function in bcCML, we tracked in vitro colony-forming ability in the presence of blocking antibodies. While inhibition of each had a significant effect on bcCML colony formation, the greatest impact was observed by blocking Sdc1 (clone 281.2, BD Pharmingen), which resulted in a four-fold loss at $0.5 \mu \mathrm{g} / \mathrm{mL}$ and eradication of nearly all colony formation at $5 \mu \mathrm{g} / \mathrm{mL}$ (Fig. 3e). Although based on this we initially prioritized functional analysis of $\mathrm{Sdc} 1$, interestingly we later discovered that syndecan and integrin $\beta_{7}$ signaling dependencies were linked.

Syndecan-1 is necessary for bcCML growth and propagation. $\mathrm{Sdc1}$ is a key mediator of cellular interactions with the microenvironment, can bind and signal in response to a variety of growth factors, and is involved in multiple adhesive processes $^{30,31}$. While syndecan expression has been recently reported on AML patient biopsies ${ }^{32}$, whether it plays a role in regulating myeloid leukemia is not known. To assess the functional role of Sdc1 in leukemia initiation through a definitive genetic model, we used Sdc1 knockout mice developed by disrupting the signaling peptide sequence within the ectodomain, thus preventing mature $\mathrm{Sdcl}$ protein from localizing at the cell surface $^{33}$ (Sdc1 ${ }^{-1-}$, Fig. 4a). Sdc1 deletion was confirmed by PCR (Supplementary Fig. 2a) and immunofluorescence staining (Fig. 4b), and its impact on normal hematopoiesis appeared to be negligible except for a minor increase in $\mathrm{Lin}^{-}$cells (Supplementary Fig. $2 \mathrm{~b}-\mathrm{h}$ ). Sdc1-1- KLS cells were infected with BCRABL and NUP98-HOXA9, and colony-forming capacity tracked. Compared to control cultures, loss of Sdc1 led to a nearly threefold reduction of bcCML colony formation (Fig. 4c). To assess the impact on LSC function we conducted a limiting dilution series in colony assays (Fig. 4d, Supplementary Fig. 3a). These data show that, at each cell number plated, bcCML colonyforming ability was reduced with Sdc1 loss. Further, with extreme limiting dilution analysis (ELDA) we found that while the stem cell frequency was $1 / 1$ for WT bcCML it was $1 / 94$ for $\mathrm{Sdc}^{-/}$ bcCML, indicating the Sdc loss triggers a 94 fold drop in stem cell capacity.

Further, transplantation of BCR-ABL/NUP98-HOXA9infected $\mathrm{Sdc1}^{-/-}$KLS cells resulted in a markedly lower (sevenfold) leukemia burden in vivo 14 days post-transplant (Fig. 4e). This impaired chimerism was not observed at an earlier time point ( 5 days), suggesting that reduced chimerism is not the result of impaired homing to the bone marrow (Supplementary 
a

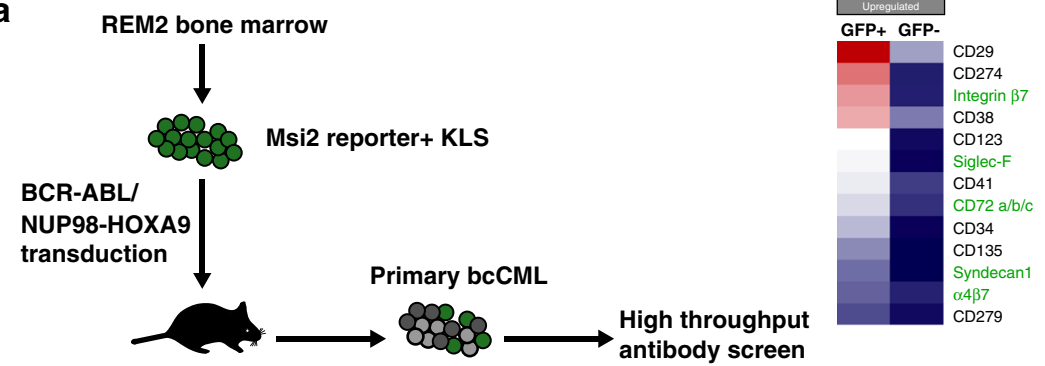

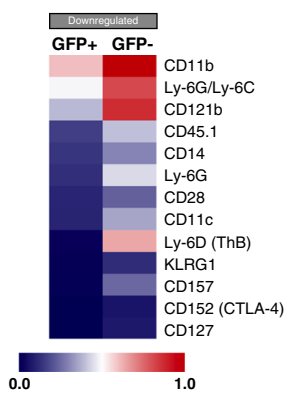

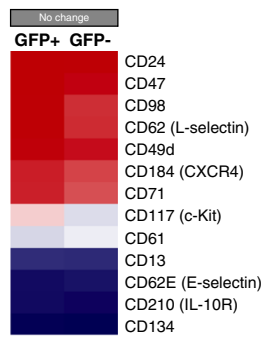

b

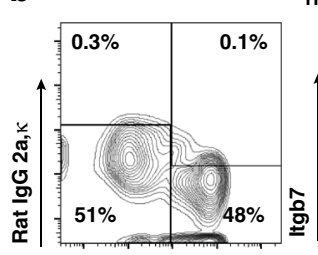

High throughput antibody screen
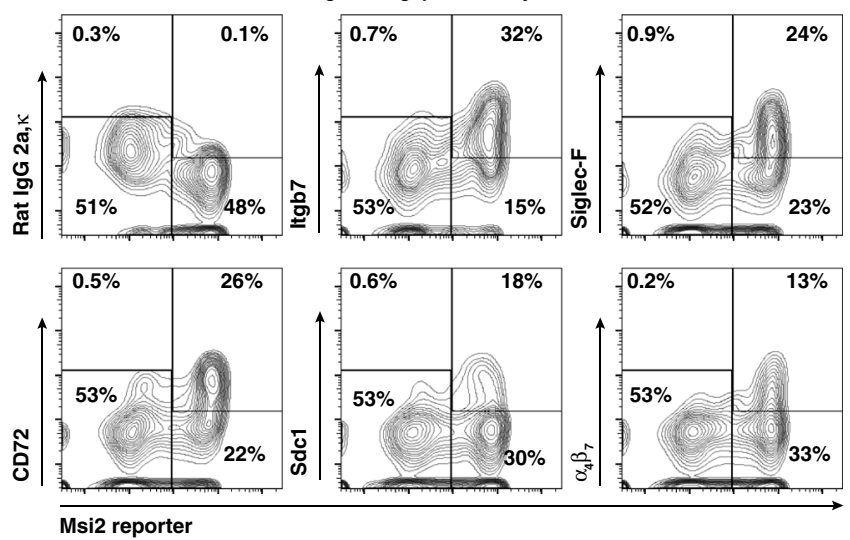

C

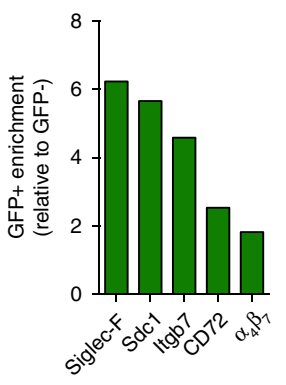

d

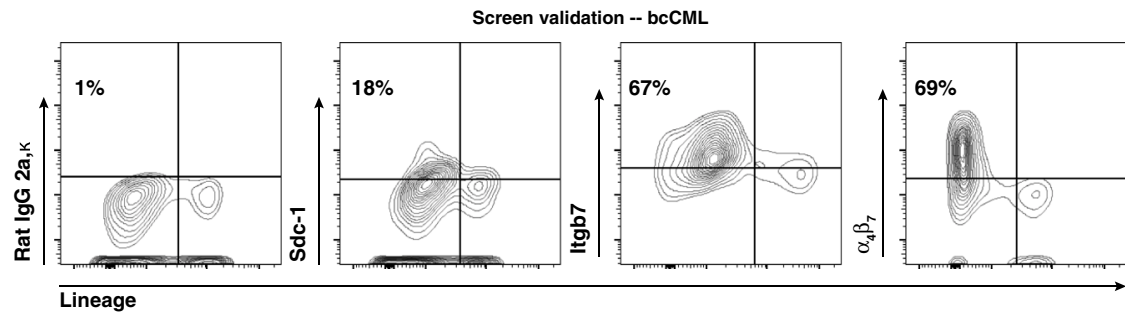

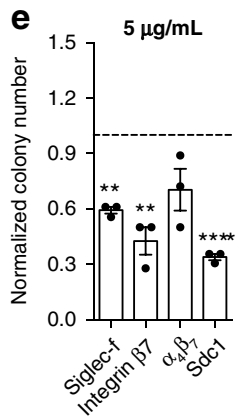

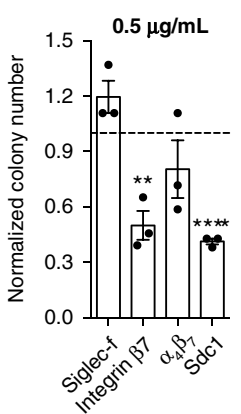

Fig. 3 High throughput screen identifies cell surface proteins with functions in Msi2-reporter bcCML cells. a Schematic illustrates the experimental approach used for FACS-based high throughput antibody screen to identify surface proteins that are differentially expressed in $\mathrm{Msi}^{+}$and $\mathrm{Msi} 2^{-}$bcCML cells. bcCML was generated from KLS cells derived from Msi2 reporter (REM2) mice. Heat maps display selected genes that are more highly expressed in $\mathrm{GFP}^{+}$bcCML cells (left panel) or GFP- bcCML cells (center panel), or that are unchanged between GFP ${ }^{+}$and GFP- bcCML cells (right panel) $(n=1$ technical replicates, representative of two biological replicates for each group). b FACS plots generated from high throughput antibody screen show increased expression of the indicated proteins on GFP+ bcCML relative to GFP- bcCML. c Enrichment of protein expression on GFP+ bcCML relative to GFP- $^{-}$bcCML based on high throughput screen ( $n=1$ technical replicates, representative of two biological replicates for each group). $\mathbf{d}$ Representative FACS plots validating expression of the indicated proteins using individual fluorescently conjugated antibodies in freshly isolated bcCML cells. e The relative number of colonies (normalized to lgG control) generated from bcCML cells in the presence of blocking antibodies against the indicated proteins at either $5 \mu \mathrm{g} / \mathrm{mL}$ (left) or $0.5 \mu \mathrm{g} / \mathrm{mL}$ (right). Significance from IgG control: $5 \mu \mathrm{g} / \mathrm{mL}$, Siglec-f ${ }^{\star \star} P=0.003$, Integrin $\beta 7^{\star \star} P=0.004$, Sdc1 ${ }^{\star \star \star \star} P<0.0001 ; 0.5$ $\mu \mathrm{g} / \mathrm{mL}$, Integrin $\beta 7^{\star \star} P=0.004$, Sdc1 ${ }^{\star \star \star \star} P<0.0001$ ( $n=3$ technical replicates, representative of three biological replicates). Two-tailed unpaired Student's $t$-tests were used to determine statistical significance. Source data are provided as a Source Data File.

Fig. 3b). We also observed reduced burden in the femur of recipient mice following intrafemoral transplantation, providing further evidence that Sdc1 loss results in an innate growth defect and not just an inability to home to the bone marrow (Fig. 4f). Most importantly, deletion of Sdc1 led to a 53\% gain in survival as measured by median survival (17 days for WT vs. 26 days for $\mathrm{Sdc1}^{-/-}$) (Fig. 4g). Since we observed a survival advantage in mice transplanted with $\mathrm{Sdc1}{ }^{-1-}$ bcCML, we tested whether Sdc1 loss can sensitize these cells to chemotherapy. Lin ${ }^{-}$bcCML cells were isolated from mice transplanted with WT or $\mathrm{Sdcl}^{-1-}$ bcCML and plated in colony-forming assays in the presence of imatinib. While WT bcCML colony numbers decreased by $50 \%$ in the presence of imatinib, Sdc1 $1^{-1-}$ bcCML colony numbers were reduced by $85 \%$ (Fig. $4 \mathrm{~h}$ ). These data suggest that Sdc1 can mediate drug resistance in bcCML LSCs, and that Sdc1 loss can sensitize bcCML cells to imatinib.

To understand the elements of Sdc1 signaling that may be important for its role in bcCML propagation, we tested the ability of wild type or a mutant Sdc1 lacking the heparan sulfate chain attachment sites to rescue the growth of $\mathrm{Sdcl}^{-1-}$ bcCML (Fig. 4i). The heparan sulfate chaifns mediate the majority of extracellular interactions of Sdc1, including matrix attachment and signaling of growth factors, whereas the Sdcl core protein is thought to potentiate inside-out integrin activation ${ }^{34,35}$. Both wild-type Sdc1 
a

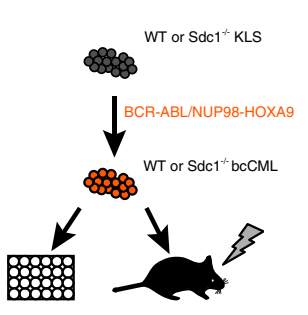

d

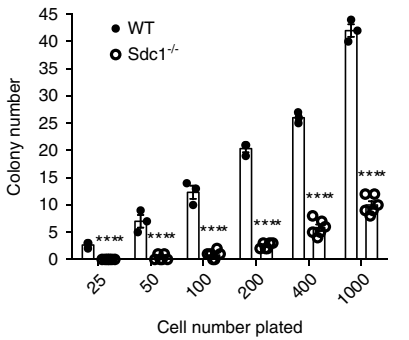

b

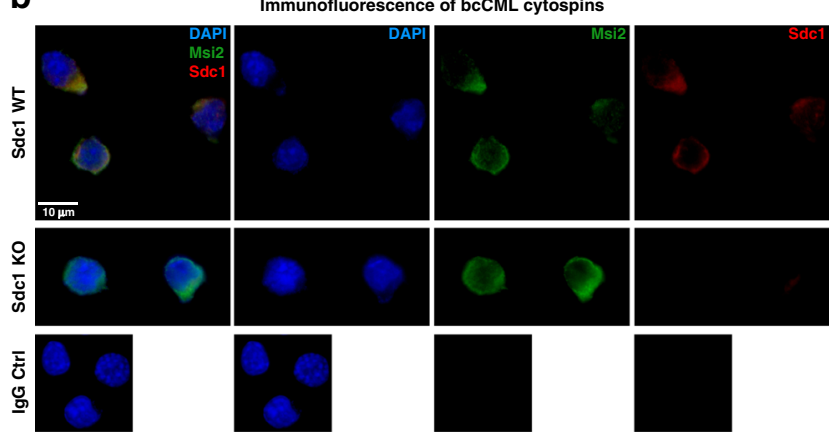

e

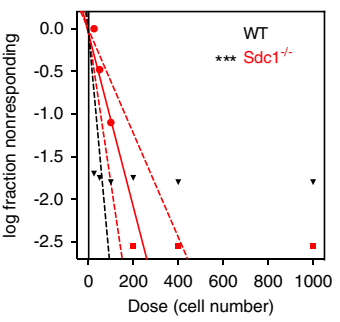

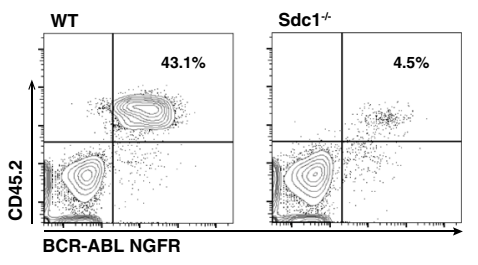
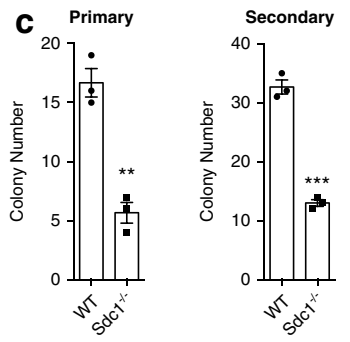

f

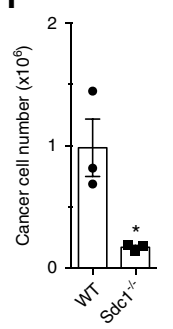

g

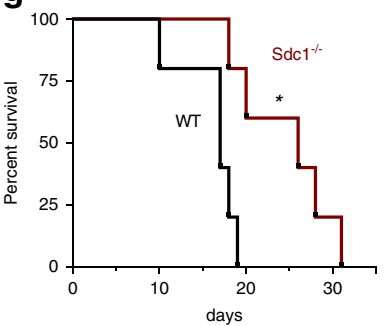

h

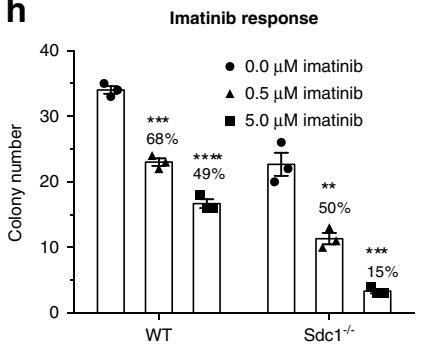

i

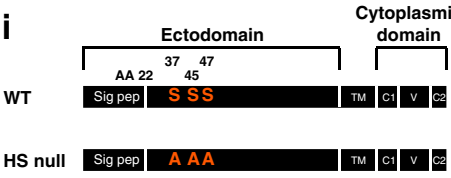

\begin{tabular}{l|l|l|l}
\hline Sig pep & A \\
\hline
\end{tabular}
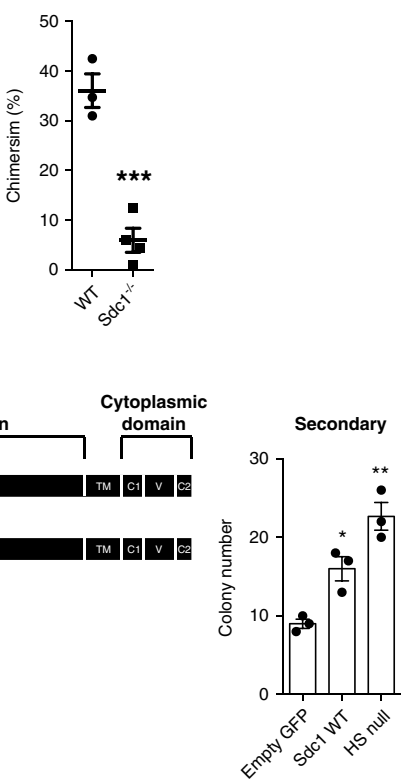

Fig. 4 Myeloid leukemia growth and propagation depend on Syndecan-1. a Approach to assess the impact of Sdc1 deletion on bcCML. b Sdc1 in WT and Sdc1 ${ }^{-/-}$bcCML (blue/DAPI, green/Msi2, red/Sdc1; 63x). c Colony formation of WT and Sdc1 ${ }^{-/-}$bcCML. ${ }^{\star \star} P=0.002$ primary and ${ }^{\star \star \star} P=$ 0.0001 secondary $\left(n=3\right.$ technical replicates from four biological replicates). d Limiting dilution assay of WT and Sdc1 ${ }^{-/}-$bcCML (LEFT). ${ }^{\star \star \star \star} P<0.0001$ ( $n=3$ technical replicates from one biological replicate for WT, $n=6$ technical replicates from two biological replicates for Sdc1 ${ }^{-/}$). Extreme limiting dilution analysis (RIGHT). Stem cell frequency: WT 1/1, Sdc1-/-1/94, ${ }^{\star \star \star} P=0.0002$. e Representative chimerism in mice transplanted with WT or Sdc1 ${ }^{-/-}$bcCML. ${ }^{\star \star \star} P=0.0007$ ( $n=3$ for WT and $n=4$ for Sdc1 ${ }^{-/}{ }^{-}$). f Intrafemoral transplant of WT or Sdc1 ${ }^{-/}-$bcCML. ${ }^{\star} P<0.03$ ( $n=3$ mice for each cohort). $\mathbf{g}$ Survival curves for WT or Sdc1 ${ }^{-/-}$bcCML. ${ }^{\star} P=0.01$ ( $n=5$ each cohort). $\mathbf{h}$ Imatinib response in WT and Sdc1 ${ }^{-/-}$bcCML. Significance from $0 \mu \mathrm{M}: \mathrm{WT}{ }^{\star \star \star} P=0.0001,{ }^{\star \star \star \star} P<0.0001$; Sdc1 ${ }^{-/-{ }^{\star \star} P} P=0.004,{ }^{\star \star \star} P=0.0004$ (\% is relative to $0 \mu \mathrm{M}$ for each group, $n=3$ technical replicates). $\mathbf{i}$ Ectopic expression of Sdc1/HS-null Sdc1 in Sdc1-/- bcCML. ${ }^{\star} P=0.01,{ }^{\star \star} P=0.002$ ( $n=3$ technical replicates from three biological replicates). Two-tailed unpaired Student's $t$-tests were used to determine statistical significance. Source data are provided as a Source Data File.

and heparan sulfate chain-null Sdc1 were able to rescue colonyforming ability in $\mathrm{Sdcl}^{-1-}$ bcCML (Fig. 4i, Supplementary Fig. 3c), suggesting a dependency on the core protein and potentially Sdc1-intergin signaling.

To test whether Sdc1 may be important for propagation of established bcCML we assessed the capacity of control and Sdc1deficient leukemia cells to propagate disease in vitro and in vivo. In vitro, shRNA knockdown of $S d c 1$ in established bcCML (Supplementary Fig. 3d) reduced primary colony formation by $\sim 3$-fold and secondary colony formation by $\sim 5$-fold (Fig. 5a, b). Given our findings that $\mathrm{Sdc1}$ is upregulated on Msi2-reporter ${ }^{+}$ cells (Fig. 3a), the population mainly responsible for driving bcCML growth, we tested whether ectopic overexpression of Sdc1 on Msi2-reporter ${ }^{-}$bcCML could enhance the growth capacity of these cells. As shown in Fig. 5c, overexpression of Sdc1 in $\mathrm{Lin}^{+}$ (Msi2-reporter ${ }^{-}$) bcCML rescued colony growth to levels similar to those of control infected $\mathrm{Lin}^{-}$(Msi2-reporter ${ }^{+}$) bcCML. These findings suggest that $\mathrm{Sdcl}$ expression can drive the acquisition of stem cell characteristics in bcCML. Control and shSdc1 bcCML cells were also transplanted into recipient mice, and disease progression monitored. shSdcl inhibition resulted in a striking reduction of leukemia burden $(\sim 7$ fold fewer transplanted cancer cells in peripheral blood) (Fig. 5d) and was significantly less lethal: mice transplanted with wild-type bcCML had a median survival of 31 days, whereas mice harboring shSdc1 bcCML lived 2.5 fold longer with a median survival of 78.5 days (Fig. 5e). Importantly, Sdc1 loss in the MLL-AF9/NRas model of AML (Supplementary Fig. 3e) drastically reduced colony formation in vitro in primary plating by $\sim 5$-fold and in secondary plating by more than 10-fold (Fig. 5f, g). These data indicate that Sdc1 is not only critical for bcCML growth, but also for other myeloid malignancies. Importantly, the dependency on syndecan signaling was conserved: thus SDC1 knockdown led to a 12 fold reduction of colony-forming ability in both a human bcCML line and a human AML line (Fig. 5h-i, Supplementary Fig. 3f); expression of SDC1 and ITGB7 in these human cell lines was confirmed by 

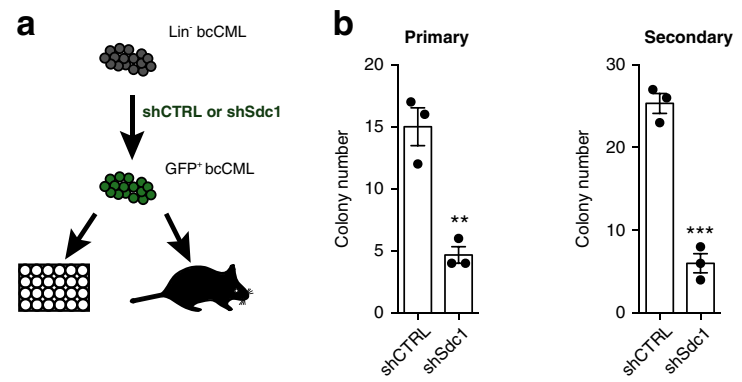

d
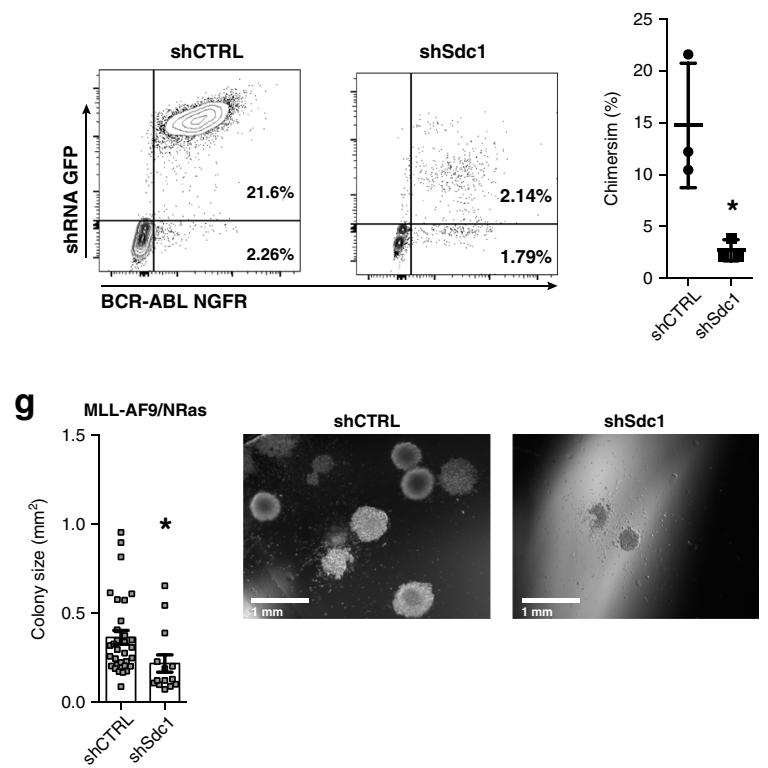

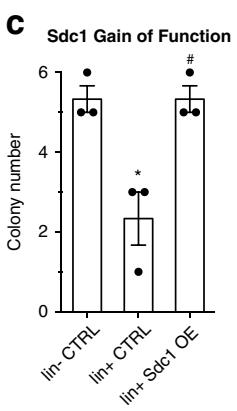

e
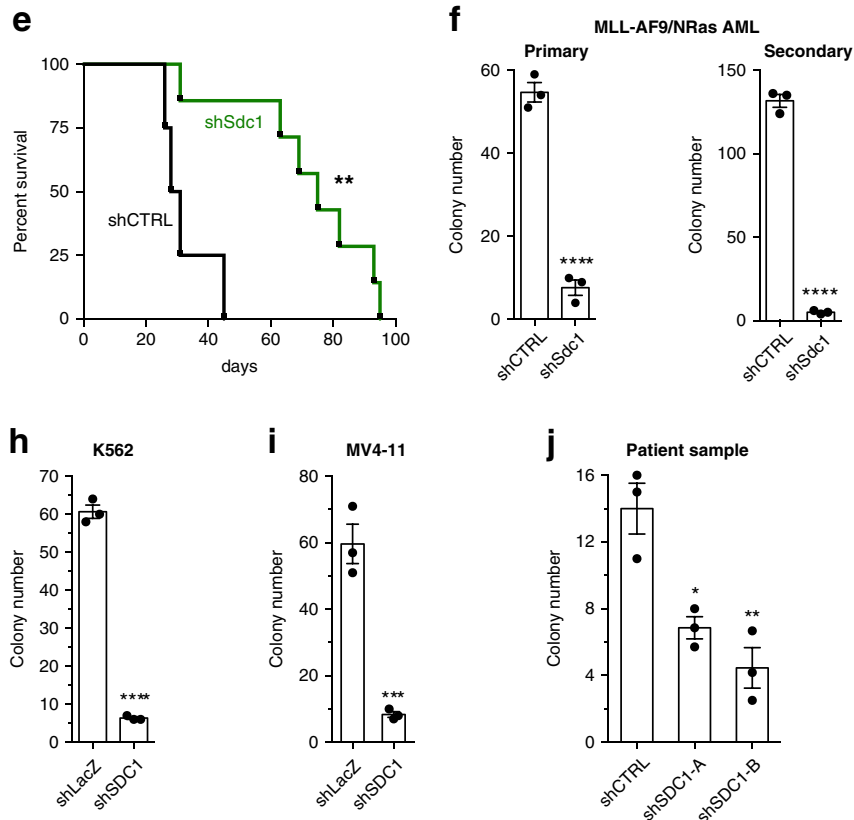

Fig. 5 Syndecan-1 loss impairs established bcCML growth in human and mouse models. a Approach to assess the impact of Sdc1 loss on established bcCML. b Colony formation of shCTRL or shSdc1 bcCML ${ }^{\star \star} P=0.003,{ }^{\star \star \star} P=0.0003(n=3$ technical replicates, representative of three biological replicates). c Impact of ectopic Sdc1 expression in $\mathrm{Lin}^{+} \mathrm{bcCML}$. ${ }^{\star} P=0.02$ from $\mathrm{Lin}^{-} \mathrm{CTRL}, \# P=0.01$ from $\mathrm{Lin}^{+} \mathrm{CTRL}(n=3$ technical replicates). d Representative chimerism of shCTRL or shSdc1 bcCML. $n=3$ mice/group. ${ }^{\star} P=0.02$. e Survival curve of mice transplanted with shCTRL or shSdc1 bcCML. ${ }^{\star \star} P=0.003$ ( $n=4$ for shCTRL and $n=7$ for shSdc1). $\mathbf{f}$ Colony formation of shCTRL or shSdc1 AML cells. ${ }^{\star \star \star \star} P<0.0001$, $(n=3$ technical replicates). $\mathbf{g}$ Impact of shSdc1 on AML. ${ }^{\star} P=0.03$ ( $n=33$ for shCTRL and $n=15$ for shSdc1). $\mathbf{h}$ Impact of shSDC1 on K562. ${ }^{\star \star \star \star} P<0.0001$ ( $n=3$ technical replicates). i Impact of shSDC1on MV4-11. Data normalized to shLacZ control. ${ }^{\star \star \star} P=0.001$ ( $n=3$ technical replicates from two biological replicates). j Impact of shSDC1 on primary patient AML. ${ }^{\star} P<0.01,{ }^{\star \star} P=0.008$ ( $n=3$ technical replicates). Two-tailed unpaired Student's $t$-tests were used to determine statistical significance. Source data are provided as a Source Data File.

qRT-PCR (Supplementary Fig. 3g). Furthermore, knockdown of $S D C 1$ in primary patient AML led to reduced colony formation as well (Fig. 5j). We should note that other syndecan family members may also contribute to myeloid leukemia, since knockdown of $S D C 2, S D C 3, S D C 4$ either individually or in combination led to significant growth inhibition (Supplementary Fig. 3h). These data collectively indicate that syndecan signaling is a key dependency of bcCML propagation and lethality, a function at least in part dependent on the core protein and independent of its heparan sulfate chains.

Loss of Sdc1 affects the spatiotemporal dynamics of bcCML cells. Because $\mathrm{Sdcl}$ can influence how cells interact with the microenvironment, we used live animal imaging to visually track its impact on the spatiotemporal dynamics of bcCML. GFPcontrol or GFP-Sdc1 knockdown bcCML cells were transplanted into dsRed recipient mice and imaged (Fig. 6a). Sdc1 knockdown was confirmed by PCR (Supplementary Fig. 4a), as well as by flow cytometry in leukemia cells to ensure that $\mathrm{GFP}^{+}$cells tracked were deficient for $\mathrm{Sdc1}$ expression (Fig. 6b). Imaging revealed that
shSdc1 bcCML recipients had nearly $50 \%$ fewer donor cells in the bone marrow relative to those receiving control bcCML (Fig. 6c, quantified in 6d), consistent with flow cytometry analysis described above. Unexpectedly, while wild-type cells were present in both the marrow space and in blood vessels at very high frequencies, the decline in Sdc1 knockdown cells was far more pronounced within the blood vessels than within the bone marrow. Specifically, control bcCML cells were more likely to be located within vessels (Fig. 6c, white arrow heads) or clustered together near vascular branching points (Fig. 6c, yellow arrow heads), while shSdcl cells could most frequently be found as isolated cells, adjacent to but outside of the vasculature (Fig. 6c, red arrow heads), resulting in a fivefold drop of Sdc1 knockdown leukemia cells in blood vessels (Fig. 6e). Further, time-lapse microscopy of individual leukemia cells in the marrow space showed a marked retardation of cell motility. Control leukemia cells traveled at $1.0 \pm 0.2 \mu \mathrm{m} / \mathrm{s}$ on average, whereas Sdc1 knockdown leukemia cells traveled at $0.37 \pm 0.05 \mu \mathrm{m} / \mathrm{s}$ (Fig. 6f; Supplementary Movies 2 and 3). Interestingly, there was a striking difference in leukemia distribution in the bone marrow and spleen, with loss of Sdc1 leading to a much steeper decline in the 
a

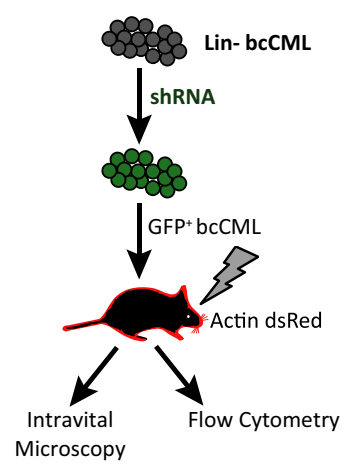

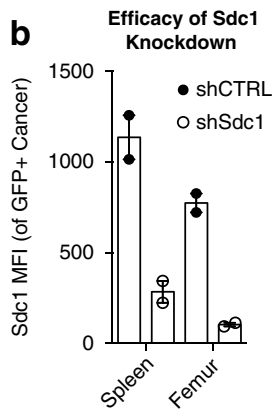

C

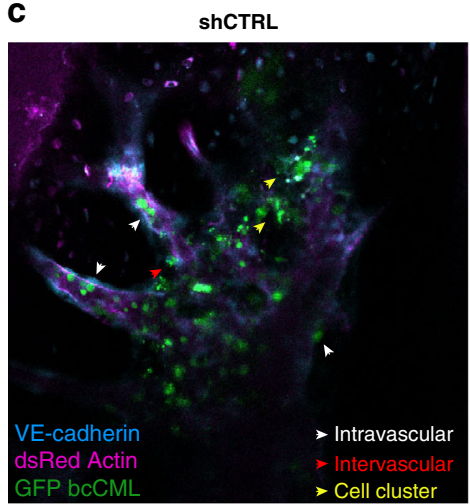

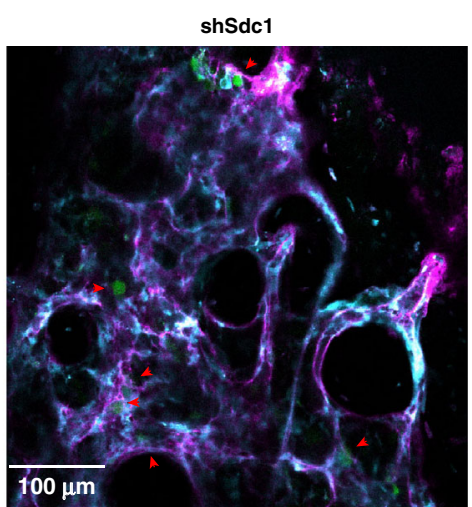
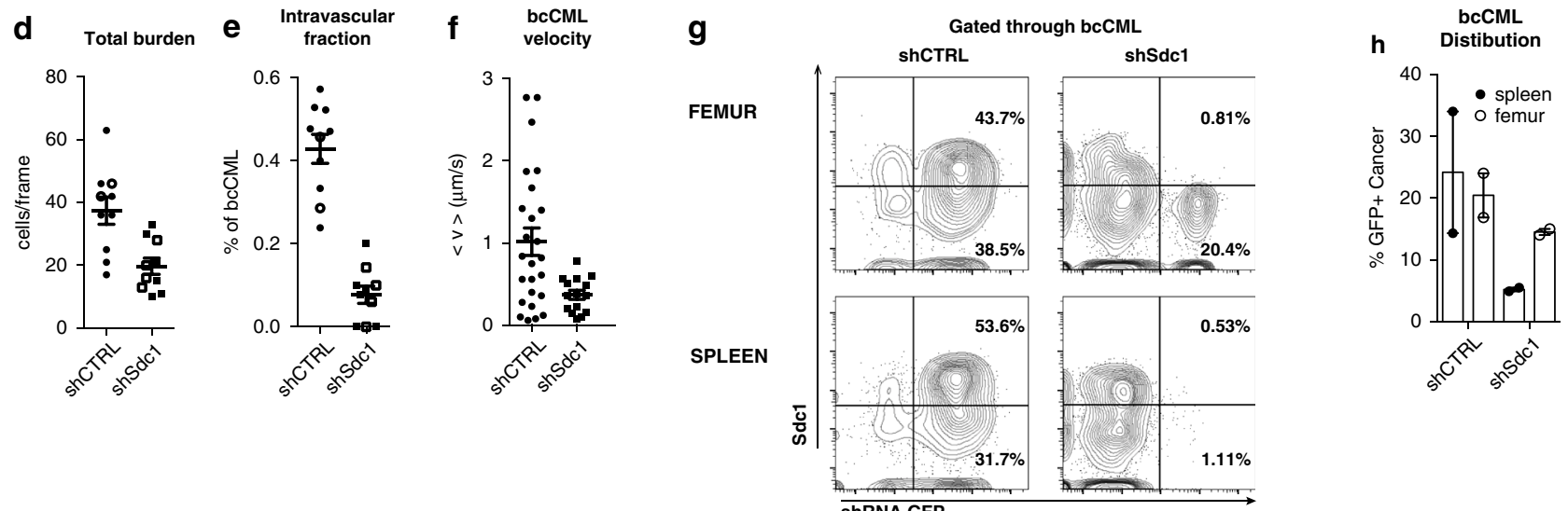

Fig. 6 Syndecan-1 controls the spatiotemporal dynamics of bcCML in vivo. a Schematic illustrates the experimental approach used to assess the impact of Sdc1 loss on the dynamics of bcCML in vivo. bcCML cells infected with shCTRL or shSdc1were transplanted into irradiated dsRed NOD SCID recipient mice. The calvarium of recipient mice was live imaged 10-15 days post-transplantation, after which the femurs and spleens were analyzed by flow cytometry. $\mathbf{b}$ Confirmation of Sdc1 knockdown in bcCML cells infected with shSdc1 as quantified as a reduction in Sdc1 MFI by flow cytometry. Significance from shCTRL within a location ( $n=2$ mice per group). c Representative live animal imaging of dsRed recipient mice transplanted with shCTRL or shSdc1 $\mathrm{bcCML}$; green $=$ shRNA vector, blue $=\mathrm{VE}$-Cadherin, magenta $=$ Actin dsRed, white arrowhead shows cells within the vasculature, red arrowhead shows cells outside of vasculature within the marrow, yellow arrowhead shows cell clusters (See also Supplementary Movies 2 and 3). d Quantification of GFP+ bcCML burden from live animal imaging of bone marrow (xyz confocal stacks, $n=2$ animals per group, open and closed symbols denote separate animals, $n=10$ fields of view per group). e Quantification of GFP + bcCML residing within vasculature determined by live animal imaging (xyz confocal stacks, $n=2$ animals per group, open and closed symbols denote separate animals, $n=10$ fields of view per group). $\mathbf{f}$ The average velocity of GFP + bcCML determined by measuring in vivo cell migration in real time (xyt confocal stacks, $n=2$ animals per group, $\geq 3$ fields of view per mouse, shCTRL $n=25$ cells, shSdc $n=$ 15 cell). $\mathbf{g}$ Representative flow cytometry plots show the distribution of bcCML in the femur and spleen of mice used in live animal imaging studies in (b-f). Percentages of shRNA-infected Sdc1+ and Sdc1- bcCML cells are indicated. $\mathbf{h}$ Quantification of tumor burden in femur and spleen of mice used in the live animal imaging studies ( $n=2$ animals per group). Two-tailed unpaired Student's $t$-tests were used to determine statistical significance. Source data are provided as a Source Data File.

spleen ( $\sim 5$-fold reduction compared to control) compared to the bone marrow ( 1.3-fold, femur, 2-fold, calvarium) (Fig. 6d, g, h). These data indicate that beyond regulating leukemia burden, Sdc1 expression may also be critical in mediating leukemia cell migration and its systemic dissemination to distant sites.

Loss of Sdc1 impairs integrin-mediated growth and migration of bcCML cells. To understand why Sdcl depletion influenced the spatiotemporal dynamics of bcCML cells, we tested if Sdc1 knockdown bcCML cells attach and migrate correctly in response to extracellular matrix cues in transwell assays and in context of endothelial cells (Fig. 7a). Sdc1 knockdown in bcCML (Supplementary Fig. 4b) showed a significant reduction in cell migration on extracellular matrix and endothelial monolayers in transwell assays compared to control bcCML (Fig. 7b, c). Cxcr4 levels on
Sdc1 ${ }^{-1-}$ bcCML cells were not impacted by Sdc1 loss, indicating that the observed migration defect is unlikely to be a result of an inherent reduced capacity to signal through Sdf-1/Cxcr4 (Supplementary Fig. 4c). Imaging of the transwells revealed that a vast majority of control bcCML cells have a polarized morphology; in contrast, shSdc1 cells displayed a significant shift towards a nonpolarized morphology (Fig. 7d). The loss in polarization may, at least in part, explain the inability of bcCML lacking Sdc1 to migrate effectively, as directional polarization allows differential attachment to extracellular matrix at the leading edge, an adhesion gradient critical to permitting movement ${ }^{36}$.

Since the live animal imaging studies showed that Sdc1 loss led to fewer bcCML cells in blood vessels, we also modeled transendothelial migration in vitro. Control and Sdc1 knockdown bcCML cells were plated on human endothelial cells (HUVECs) that had been grown on fibronectin-coated polyacrylamide gels 

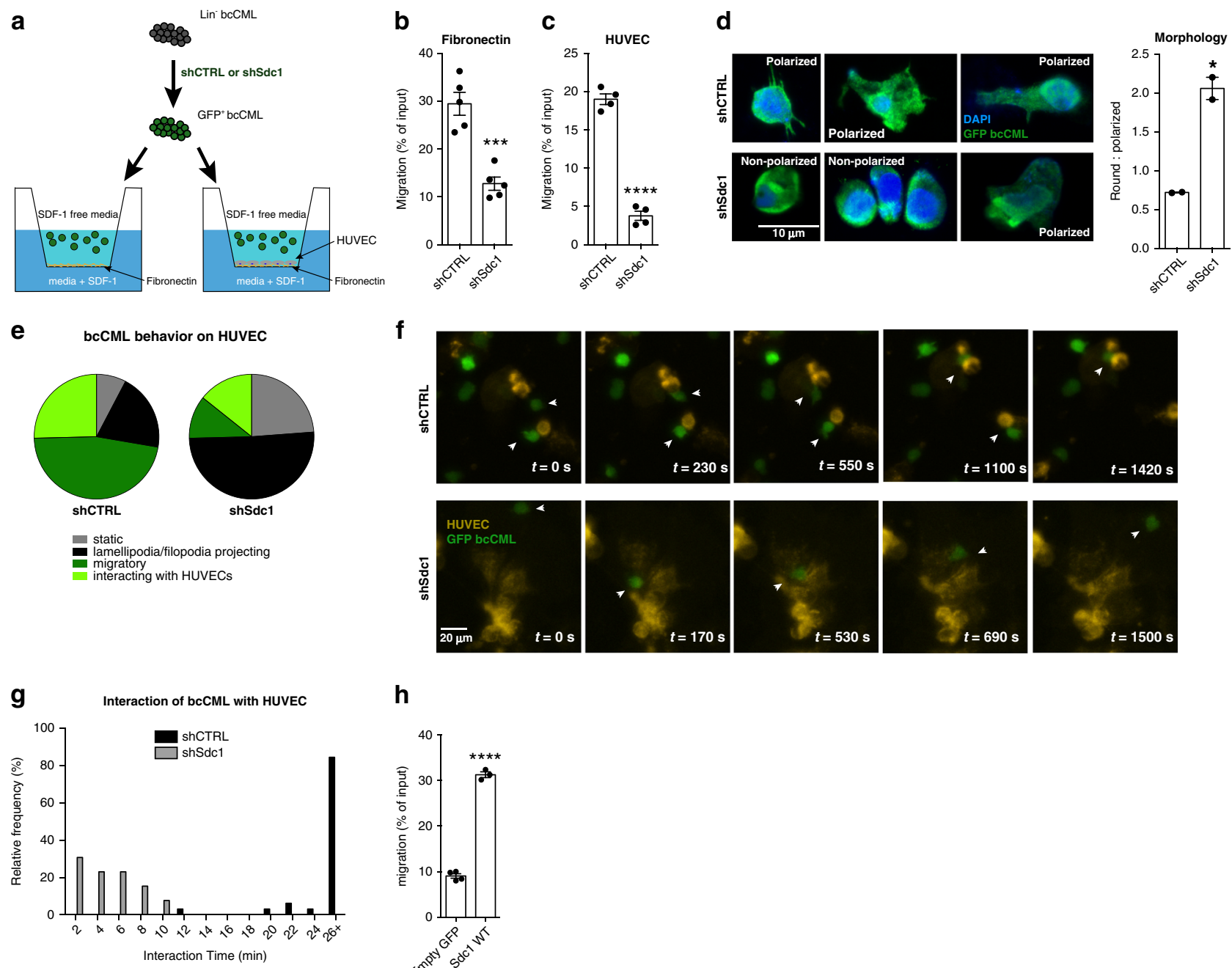

h

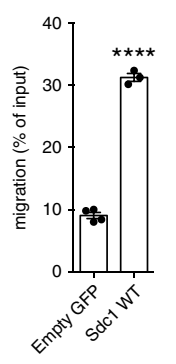

Fig. 7 Syndecan-1 mediates cell migration. a Schematic of transwell migration assay across either FN or FN+ HUVEC monolayer. b SDF-1 induced migration of shCTRL or shSdc1 bcCML cells across fibronectin-coated transwell filters. ${ }^{\star \star \star} P=0.0003$ ( $n=3$ technical replicates, representative of four biological replicates). c SDF-1 induced migration of shCTRL or shSdc1 bcCML cells across HUVEC monolayers seeded on fibronectin-coated transwell filters. ${ }^{\star \star \star \star} P<0.0001$ ( $n=4$ technical replicates, representative of two biological replicates). d Representative immunofluorescence of shCTRL or shSdc1 bcCML after $4 \mathrm{~h}$ incubation on fibronectin-coated transwell filters; green = shRNA vector, blue = DAPI. Quantification of polarized and non-polarized shCTRL or shSdc1 cells. ${ }^{\star} P=0.01$ ( $n=3$ biological replicates, $>30$ cells per condition). e-g Characterization of bcCML behavior when interacting with HUVECs on fibronectin-coated polyacrylamide gels $(8 \mathrm{kPa})$. e Cell behaviors are stratified as: completely immobile (gray = static); immobile, but sensing its surroundings by extending lamellipodia and or filopodia (black = lamellipodia/filiopodia projecting); migratory, but not interacting with HUVECs (dark green $=$ migratory); migratory and sustaining prolonged interactions with HUVECs (light green = interacting with HUVECs) ( $n>10$ cells per field of view for four fields across two independent experiments). $\mathbf{f}$ Representative time-lapse images demonstrating bcCML cell behavior in proximity to HUVECs on fibronectin-coated polyacrylamide gels (See also Supplementary Movies 4 and 5). g Duration of interactions between bcCML and HUVECs. ( $n=33$ events for shCTRL and $n=13$ events for shSdc1). h SDF-1 induced migration of Sdc1-/- bcCML cells with empty control or wild-type Sdc1 ectopically expressed across HUVEC monolayers seeded on fibronectin-coated transwell filters. ${ }^{\star \star \star \star} P<0.0001$ ( $n=4$ technical replicates). Two-tailed unpaired Student's $t$-tests were used to determine statistical significance. Source data are provided as a Source Data File.

with a stiffness comparable to bone marrow $(8 \mathrm{kPa})$. Real-time imaging revealed that bcCML cells lacking Sdc1 were far less motile than control cells (Fig. 7e, f; Supplementary Movie 4). While control cells showed a directed movement towards the endothelial cells and displayed long temporal associations (Fig. 7g), leukemic cells lacking Sdc1 were less motile and non directed in their motion, and remained largely non-interactive with the endothelial cells (Fig. 7e-g; Supplementary Movie 4). Further, ectopic expression of wild-type Sdc1 in Sdc1 ${ }^{-1-}$ bcCML (Supplementary Fig. 4d, e) rescued cancer cell migration to nearnormal levels (Fig. 7h), indicating a direct role for syndecan in bcCML movement. Collectively, these data highlight a fundamental functional relationship between Sdcl expression and
bcCML migration and localization, and raise the exciting possibility that disabling Sdc1 function could be implemented as a strategy to reduce tumor burden, as well as systemic dissemination.

In an attempt to further understand the impact that Sdc1 loss has on bcCML growth and migration we conducted an RNAseq analysis of WT bcCML versus Sdc1 ${ }^{-1-}$ bcCML cells (Fig. 8a). The principal component analysis showed that $\mathrm{Sdcl}^{-1-}$ bcCML cells were distinct from WT bcCML cells at a global transcriptional level (Supplementary Fig. 5a) and were defined by the differential expression of hundreds of genes (Supplementary Fig. 5b). Gene set enrichment analysis of the resultant data identified a strong impact on pathways falling within the categories of oncogenic 
a

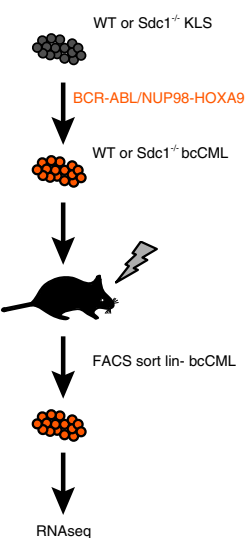

b

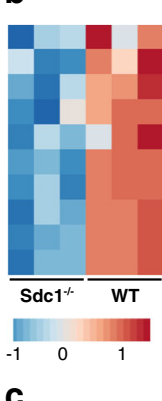

Oncogenic signatures

KREPPEL_CD99_TARGETS_UP BUDHU LIVER CANCER METASTASIS UP WANG_ESOPHAGUS CANCER PROGRESSION UP SMID_BREAST_CANCER_RELAPSE_IN PLEURA_UP SMID_BREAST_CANCER_RELAPSE_IN_PLEURA_UP
RAMJAUN_APOPTOSIS_BY_TGFB1_VIA_SMAD4_UP STEARMAN_TUMOR_FIELD_EFFECT_UP SWEET_KRAS_ONCOGENIC_SIGNATURE ONO AML1 TARGETS UP

IIZUKA LIVER CANCER_PROGRESSION_L1_G1 UP MISHRA_CARCINOMA_ASSOCIATED_FIBROBLAST_UP

C

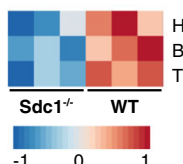

Relapse/Resistance signatures

HOLLEMAN_ASPARAGINASE_RESISTANCE_B_ALL_UP BARRIER_CANCER_RELAPSE_NORMAL_SAMPLE_UP TSUNODA_CISPLATIN_RESISTANCE_UP d

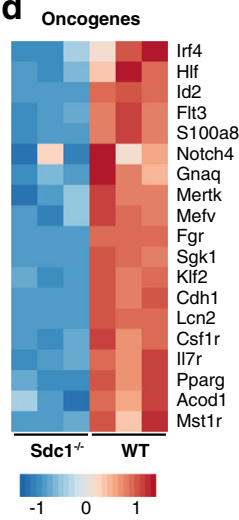

e

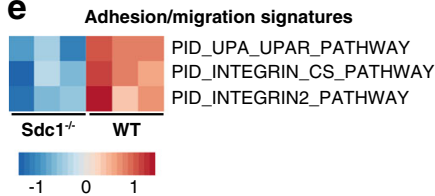

f Integrins

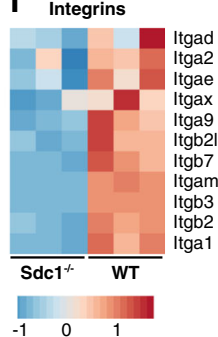

g

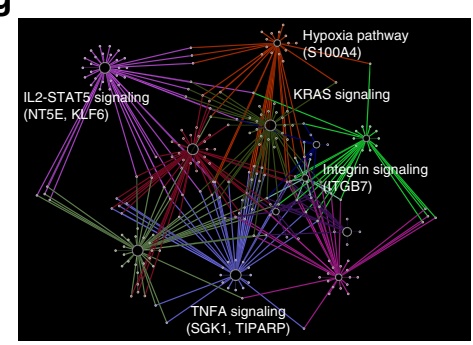

Fig. 8 Syndecan-1 loss disrupts growth and migration programs. a Schematic of RNAseq workflow. b GSEA analysis of WT vs. Sdc1-/- bcCML RNAseq showing impact on oncogenic pathways. c GSEA analysis of WT vs. Sdc1-/- bcCML RNAseq showing impact on drug resistance/relapse pathways. d Heat map of selected genes falling within the GSEA oncogenic and resistance/relapse pathways. e GSEA analysis of WT vs. Sdc1-/- bcCML RNAseq showing impact on adhesion/migration pathways. $\mathbf{f}$ Heat map of integrin gene expression in Sdc1-/- bcCML falling within the GSEA adhesion/migration pathways. $\mathbf{g}$ Network analysis of genes downregulated in $\mathrm{Sdc} 1^{-/-}$bcCML by RNAseq crossed to a recently published bcCML CRISPR screen.

(Fig. 8b) and resistance/relapse (Fig. 8c) that could explain the observed growth defect. Included within these pathways are oncogenic signals such as Flt3, Klf2, and Notch4 (Fig. 8d). The migration defect accompanying Sdc1 loss could be explained by the impact on adhesion/migration pathways (Fig. 8e) particularly integrins such as such as Itgb2, Itgb3, Itga9, and Itgax (Fig. 8f). Interestingly, Itga2, Itga $9, \operatorname{Itg} \beta 5$, and $\operatorname{Itg} \beta 7$ have been shown to control both growth and migration and may link the observed migration and growth defects. We then crossed the $\mathrm{Sdcl}^{-/}$ bcCML downregulated genes with a recent bcCML CRISPR screen performed in our laboratory ${ }^{37}$. The resulting 111 common genes were then used for Cytoscape enrichment analysis and visualization. This mapping demonstrates the interconnectedness of integrin signaling and oncogenic/survival pathways (Fig. 8g) and the dependence these programs have on Sdc1 in bcCML.

To further understand the mechanism by which Sdc1 impacts bcCML growth and migration we focused on integrin $\beta_{7}(\operatorname{Itg} \beta 7)$ as it was identified in our RNAseq analysis and was highly enriched on $\mathrm{Msi} 2^{+}$bcCML cells in the high throughput screen (Fig. 3a-c). Its functional inhibition impaired growth of bcCML colony formation (Figs. 3e, 9a and Supplementary Fig. 5c) and bcCML migration on fibronectin-coated filters (Fig. 9b). While the fact that Itg $\beta 7$ loss phenocopied Sdc1 loss was consistent with the signals being in a hierarchy, we directly tested this possibility by determining if the absence of Sdc1 function specifically impaired Itg $\beta 7$ function. To this end, we repeated the transwell migration experiment using filters coated with the Itg $\beta 7$ specific ligand MAdCAM-1, and observed a significant decrease in migration with Sdc1 loss (Fig. 9c). In addition, we performed a soluble ligand binding assay with WT or $\mathrm{Sdcl}^{-1-}$ bcCML cells using MAdCAM-1 or VCAM-1, an integrin $\alpha_{4} \beta_{1}$ ligand. While Sdc1 deletion impaired binding of bcCML cells to MAdCAM-1 by nearly 3-fold (Fig. 9d), binding to the control VCAM-1 was not affected (Fig. 9e). These data confirm that $\mathrm{Sdc1}$ is necessary for the normal function of $\operatorname{Itg} \beta 7$ and that the coordinated action of $\mathrm{Sdcl}$ and $\operatorname{Itg} \beta 7$ is a key regulatory element in bcCML. We should note that shSdcl knockdown did reduce Itg $\beta 7$ surface expression by $15 \%$ (Supplementary Fig. $5 \mathrm{~d}$ ); because the majority of integrin surface expression was present even with Sdc1 loss, it may suggest the defects observed are more likely to be due to impaired integrin potentiation. While overexpression of Sdc1 or Itg $\beta 7$ could rescue the colony-forming defect observed in $\mathrm{Sdcl}^{-}-\mathrm{bcCML}$ to some extent, the two together were more effective in triggering colony growth, suggesting a synergistic mechanism of action (Fig. 9f, Supplementary Fig. 5e). Further, treatment with an Itg $\beta 7$ antagonist (firategrast) impaired WT bcCML colony formation but did not impact $\mathrm{Sdcl}^{-/}$bcCML colony growth (Supplementary Fig. 5f). This finding suggests that in the context of Sdc1 loss, further antagonism of Itg $\beta 7$ has no additional impact on colony formation because Sdc1-Itg $\beta 7$ signaling is already impaired, while WT bcCML has intact Sdc1-Itg $\beta 7$ signaling and is susceptible to Itg $\beta 7$ antagonism. Likewise, defects in transwell migration of $\mathrm{Sdc1} 1^{-1-}$ bcCML on FN coated filters could be rescued with wild-type $\mathrm{Sdc1}$ to near-normal levels while rescue of $\mathrm{Sdc1} 1^{-/-}$bcCML with $\operatorname{Itg} \beta 7$ increased migration above that of WT control bcCML (Fig. 9g). Finally, while the HS-null Sdc1 mutant could rescue colony formation of $\mathrm{Sdcl}^{-/}$bcCML (Fig. 4i), the HS-null mutant was unable to rescue the migratory defect (Supplementary Fig. 5g). These data may indicate that the HS chains are critical to "capturing" the cytokine (SDF-1 in this case) or in interacting with the microenvironment to facilitate migration. 

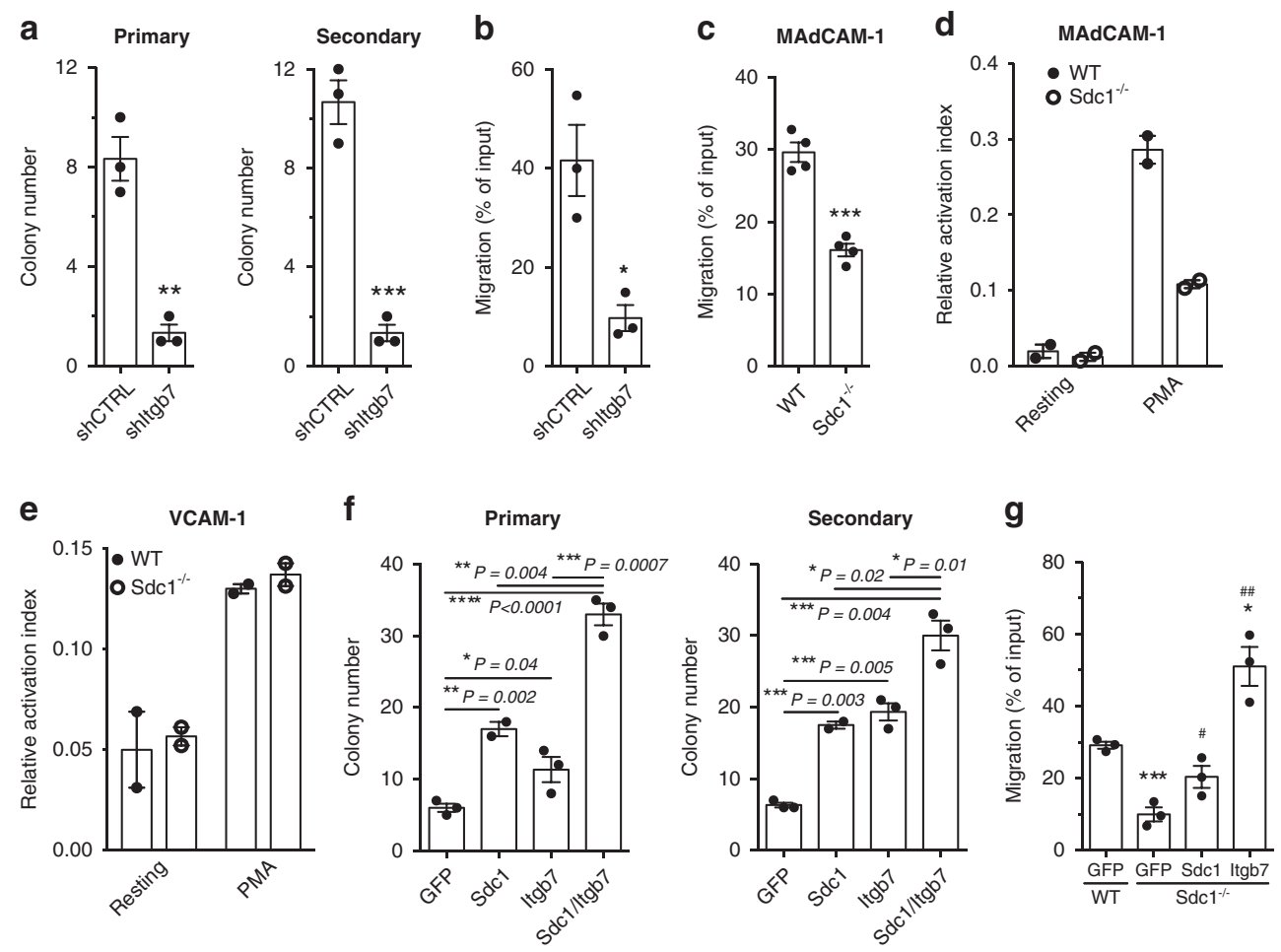

Fig. 9 Syndecan-1 loss impairs integrin $\boldsymbol{\beta} \mathbf{7}$ activity. a Number of colonies generated from shCTRL or shltg $\beta 7$ knockdown of bcCML cells after the primary (left panel) and secondary plating (right panel). ${ }^{\star \star} P=0.001,{ }^{\star \star \star} P=0.0006(n=3$ technical replicates, representative of 3 biological replicates). $\mathbf{b}$ SDF- 1 induced migration of shCTRL or shltg $\beta 7$ bcCML cells across HUVEC monolayers seeded on fibronectin-coated transwell filters. ${ }^{\star} P=0.01(n=4$ technical replicates, representative of two biological replicates). c SDF-1 induced migration of WT or Sdc1 ${ }^{-/}$bcCML cells across MAdCAM coated transwell filters. ${ }^{\star \star \star} P=0.0002$ ( $n=4$ technical replicates, representative of two biological replicates). $\mathbf{d}$ Soluble ligand binding assay with WT and Sdc1-/- bcCML and the Itg $\beta 7$ ligand, MAdCAM-1, either resting or stimulated with $200 \mathrm{nM}$ phorbol myristate acetate (PMA). Data are normalized to non-physiological stimulation with $1 \mathrm{mM} \mathrm{Mn}^{2+}$ ( $n=2$ technical replicates, representative of two biological replicates). e Soluble ligand binding assay with WT and Sdc1-/- bcCML and the Itg $\beta 1$ ligand, VCAM-1, either resting or stimulated with $200 \mathrm{nM} \mathrm{PMA}$. Data are normalized to non-physiological stimulation with $1 \mathrm{mM} \mathrm{Mn}{ }^{2+}$. $(n=2$ technical replicates, representative of two biological replicates). $\mathbf{f}$ Rescue of Sdc1-/- primary and secondary colony formation by ectopic expression of either empty GFP control, wild-type Sdc1, Itg $\beta 7$ or Sdc1 + Itg $\beta 7$. ( $n=3$ technical replicates, representative of three biological replicates). $\mathbf{g}$ Rescue of $\mathrm{Sdc}^{-1-}$ migration on FN coated filters by ectopic expression of either empty GFP control, wild-type Sdc1, or $\operatorname{Itg} \beta 7 .{ }^{\star} P=0.02,{ }^{\star \star \star} P=0.0009, \# P=0.04$, $\# \# P=0.002$. ${ }^{*}$ Significance from WT bcCML + empty GFP, \#significance from Sdc1 ${ }^{-/}+$empty GFP ( $n=3$ technical replicates, representative of three biological replicates). Two-tailed unpaired Student's $t$-tests were used to determine statistical significance. Source data are provided as a Source Data File.

\section{Discussion}

Past studies have predominantly used cell surface markers such as CD133 to identify and isolate tumor cells with stem cell-like properties. Although these efforts have enhanced our understanding of tumor stem cell populations, surface markers cannot be effectively used to track cells in vivo and would require complex antibody panels to assess residual disease. In recent years, the ability to identify and track tumor-propagating cells in solid cancers has been greatly improved through the use of reporter mice. For example, in studies of glioblastoma multiforme, Nestin-GFP mice enabled identification of rare Nestinexpressing tumor cells that exhibit many cancer stem cell-like characteristics and are chemo-resistant ${ }^{38}$. Similarly, SOX2-GFP reporter mice were used to identify tumor-propagating cells in skin squamous-cell carcinoma ${ }^{39}$, and an ERG +85 reporter has recently been used to identify LSCs within heterogeneous AML samples ${ }^{40}$. Importantly, the Msi2-reporter mouse we developed may be valuable not only for identifying LSCs but for a broader range of aggressive cancers with highly upregulated Msi2 expression, such as hepatocellular carcinoma, glioblastoma and breast cancer ${ }^{18,41}$. The ability to identify therapy-resistant tumorpropagating cells in vivo provides a proof of principle for improving approaches to therapy. For example, following leukemia treatments via cytotoxic and targeted therapies, or radiation prior to allogeneic transplant, image-based detection of therapyresistant cells could, in the long term, allow areas of residual disease to be identified and targeted with alternative strategies or higher localized doses to improve efficacy and minimize collateral tissue damage.

The screen we performed to identify differentially expressed surface proteins on $\mathrm{Msi2} 2^{+}$leukemia cells led to the identification of $\mathrm{Sdcl}$ as a key regulator of bcCML growth and propagation. Prior work has implicated Sdc1 in solid cancers such as breast cancer ${ }^{42,43}$ and in multiple myeloma ${ }^{44-46}$. Our discovery that Sdcl plays a role in bcCML indicates that it may in fact be a core regulatory mechanism for leukemias as well, and suggests that interactions with their niche are a critical source of support for leukemia cells.

A particularly exciting aspect of our studies is the finding that $\mathrm{Sdcl}$ is required for dissemination of bcCML stem cells. Although mechanisms of metastasis in solid tumors have been widely studied, the basis of cell migration in leukemia is generally less understood. Past studies have focused on bone marrow homing, and implicated SDF-1/CXCR4 in B-ALL homing in vivo ${ }^{47,48}$, and CD44 49,50 in CML and de novo AML homing. Our work is distinct in exploring how leukemia cells migrate away from bone marrow niches, and open ground in our understanding of the molecular basis of bcCML migration by demonstrating that Sdc1 
loss leads to a dramatic shift in the spatio-temporal dynamics of bcCML, specifically impairing transit velocity, intravascular frequency and effective colonization of distant sites. Further, the fact that Sdc1 loss impaired adhesion and migration suggests that Sdc1 may control widespread dissemination by facilitating attachment and locomotion on matrix proteins. Syndecans have been shown to have the ability to directly bind matrix proteins such as vitronectin, fibronectin, laminin, and collagens through their heparan sulfate chains ${ }^{51-53}$, and to modulate the ability of cells to interact with the ECM through regulation of integrin activities $^{54,55}$. For example, in multiple myeloma, heparanase trimming of the Sdc1 HS chains allows $\mathrm{MMP}^{-}$9-mediated shedding of Sdc1. The shed Sdc1 can then couple VEGFR2 to integrin $\alpha_{4} \beta_{1}$, resulting in an invasive phenotype ${ }^{44}$. In that context our data show that $\operatorname{Itg} \beta 7$ is not only enriched on $\mathrm{Msi}^{+}$ leukemic bcCML cells but that $\mathrm{Sdc} 1$ directly regulates its function. While Itg $\beta 7$ is expressed in lymphomas in addition to $\mathrm{AML}^{56}$ its function in hematologic malignancies was unknown. Thus our data provide insight into the role of $\operatorname{Itg} \beta 7$ in myeloid leukemia, and suggest this could be an exciting avenue of future research. This also provides further support to emerging evidence that Itg $\beta 1$ activity, and CD98, which amplifies integrin signaling, can regulate myeloid leukemia ${ }^{57-59}$. The current work adds further insight not only in identifying $\operatorname{Itg} \beta 7$ as an element in myeloid leukemia but also in defining a role for Sdc1 in coordinating integrin activity in hematologic malignancies. Interestingly, a potential link between $S \mathrm{dc} 1, \operatorname{Itg} \beta 7$, and Msi2 may be found in Msi2's role as an RNA binding protein. The Msi2 consensus binding sequence $\left(\mathrm{G} / \mathrm{AU}_{1-3} \mathrm{AGU}\right)^{60}$ has been studied in detail in human, mouse, and drosophila ${ }^{61}$. Analysis of the $3^{\prime}$-UTRs of Sdc1 and $\operatorname{Itg} \beta 7$ for Msi2 binding sites revealed multiple instances of at least the core element in each transcript (Supplementary Fig. 5h). Therefore, it is possible that Msi2 can directly influence the expression of Sdc1 and $\operatorname{Itg} \beta 7$. This could be in addition to regulation of the Sdc1 promoter $^{62}$ by the proximal promoter and under the control of members of the Sp1 transcription factor family. While a role for syndecans, integrins, and adhesive signaling has been investigated in the migration and growth of solid cancers $^{34,54,63}$, our data provide a perspective on liquid tumors showing an unexpected importance of adhesion events in leukemia growth and dissemination.

The data discussed here collectively highlight the Sdc1-Itg $\beta 7$ signaling axis as a key regulatory control point in sensing microenvironmental cues to regulate bcCML growth and dissemination, and suggest that blocking either $S \mathrm{dc} 1$ or $\operatorname{Itg} \beta 37$ could weaken such interactions and make leukemic cells more vulnerable to effective targeting. Development of blocking $\mathrm{mAbs}$ may be effective in exploiting this vulnerability; certainly the fact that SDC1 inhibition also blocked human bcCML growth provides a strong rationale for further pursuing the possibility of targeting Sdc1 in a clinical setting. An anti-Sdc1 mAb conjugated to chemotherapeutic agents has been developed and is currently in trials for multiple myeloma ${ }^{45}$. Further, a novel CAR $\mathrm{T}$ cell targeting Itg $\beta 7$ has shown encouraging results in eliminating cancer cells in a murine multiple myeloma model ${ }^{64}$. In this context, our data that $S d c 1$ and $\operatorname{Itg} \beta 7$ are highly expressed in bcCML raises the possibility that similar agents could serve as approaches to eradicating LSCs and improving bcCML control.

\section{Methods}

Mice. All animal experiments were performed according to protocols approved by the University of California San Diego Institutional Animal Care and Use Committee. Mice were bred and maintained in the animal care facilities at the University of California San Diego. The following mice were used: B6-CD45.2 and B6CD45.1 (Strain: B6.SJL-Ptprc ${ }^{a} P e p c^{b} /$ BoyJ); NSG mice (Strain: NOD.Cg-Prkdc scid $I l 2 r g^{t m 1} \mathrm{Wjil}_{\mathrm{SzJ}}$ ); Actin-dsRed mice (also referred as Actb-dsRed. T3 mice, (Strain:
} NOD.Cg-Prkd ${ }^{\text {scid }}$ Tg(CAG-DsRed*MST)1Nagy/KupwJ). REM2 (Msi2 ${ }^{+/ G P P}$ ) reporter mice have been described previously, ${ }^{65}$. $\mathrm{Sdcl}^{-1-}$ mice have been previously described ${ }^{33}$. All mice were $8-16$ weeks of age. Mice were bred and maintained in the animal care facilities at the University of California, San Diego. All animal experiments were performed according to protocols approved by the University of California, San Diego Institutional Animal Care and Use Committee.

Generation and analysis of leukemic mice. For BCR-ABL1/NUP98-HOXA9driven blast crisis CML (bcCML), Mll-AF9-driven AML, bone marrow-derived KLS cells were isolated and sorted from REM2 mice, CD45.2 B6, or Sdc1 ${ }^{-/}$mice. All sorted cells were cultured overnight in X-Vivo15 media (Lonza) supplemented with $50 \mu \mathrm{M} 2$-mercaptoethanol, $10 \%$ (vol/vol) fetal bovine serum, SCF (100 ng/ml, R\&D Systems) and TPO (20 ng/ml, R\&D Systems). Cells were retrovirally infected with MSCV-BCR-ABL-IRES-NGFR and MSCV-NUP98-HOXA9-IRES-huCD2 to generate bcCML; MSCV-MLL-AF9a-IRES-NGFR and MSCV-NRas G12V_IREShuCD2 to generate MLL-AF9-driven AML. Subsequently, cells were harvested $48 \mathrm{~h}$ after infection. For bcCML primary transplants, BCR-ABL ${ }^{+} / \mathrm{NUP} 98 \mathrm{HOXA} 9^{+}$cells were transplanted retro-orbitally into cohorts of sub-lethally (6 Gy) irradiated CD45.1 mice. For bcCML secondary transplants, BCR-ABL ${ }^{+} /$NUP98-HOXA9 $^{+}$ spleen cells recovered from terminally ill primary recipients were sorted and either $5000 \mathrm{GFP}^{+}$or $\mathrm{GFP}^{-}$leukemia cells were transplanted into sub-lethally $(6 \mathrm{~Gy})$ irradiated secondary recipients. For MLL-AF9 primary transplants, MLL-AF9+/ $\mathrm{NRas}^{+}$cells were transplanted retro-orbitally into cohorts of sub-lethally (6 Gy) irradiated CD45.1 mice. For MLL secondary transplants, MLL-AF9 ${ }^{+} / \mathrm{NRas}^{+}$spleen cells recovered from terminally ill primary recipients were sorted and either 500 $\mathrm{GFP}^{+}$or $\mathrm{GFP}^{-}$leukemia cells were transplanted into sub-lethally (6 Gy) irradiated secondary recipients. Cell isolation, culture, infection and primary and secondary transplantation assays for $M L L$-driven leukemia were performed as previously described $^{65}$. Disease mice were analyzed as previously described ${ }^{66}$.

In vitro radiation and imatinib treatment. Bulk blast crisis CML cells recovered from the spleen of terminally ill recipient mice that were initially transplanted with $\mathrm{BCR}^{-\mathrm{ABL}^{+} / \mathrm{NUP} 8 \text {-HOXA9 }}{ }^{+}$KLS cells isolated from REM2 mice were either irradiated $(0,5$, or $10 \mathrm{~Gy})$ in PBS with glucose and cultured in X-Vivo15 media (Lonza) supplemented with $50 \mu \mathrm{M} 2$-mercaptoethanol, $10 \%$ (vol/vol) fetal bovine serum, SCF (100 ng/ml, R\&D Systems) and TPO (20 ng/ml, R\&D Systems) for $7 \mathrm{~h}$ or treated with imatinib $(0.5$ or $5 \mu \mathrm{M})$ or control DMSO for $7 \mathrm{~h}$ in X-Vivol 5 media (Lonza) supplemented with $50 \mu \mathrm{M}$ 2-mercaptoethanol, 10\% (vol/vol) fetal bovine serum, SCF (100 ng/ml, R\&D Systems) and TPO (20 ng/ml, R\&D Systems). Cells were then washed and stained with antibodies against lineage markers. Apoptosis assays were performed by staining cells with Annexin-V (BD Pharmingen).

In vitro methylcellulose colony formation assays. For methylcellulose assays performed with blast crisis CML (bcCML) cells, BCR-ABL/NUP98-HOXA9-driven leukemia was generated using KLS cells isolated from B6-CD45.2 or Msi2-reporter mice. Primary bcCML cells were fractionated based on GFP expression $\left(\mathrm{GFP}^{+}\right.$or $\mathrm{GFP}^{-}$) and 250 cells from each fraction were plated in methylcellulose media: Iscove's modified medium-based methylcellulose medium (Methocult GM M3434, StemCell Technologies). For methylcellulose assays performed with lineagenegative bcCML cells, lineage-negative primary bcCML cells were fractionated based on GFP expression $\left(\mathrm{GFP}^{+}\right.$and $\left.\mathrm{GFP}^{-}\right)$and 500 cells from each fraction were plated. For serial plating, 500 cells derived from primary colonies were re-plated in fresh methylcellulose media. For serial plating, 5000 cells derived from primary colonies were re-plated. For methylcellulose assays performed with MLL-AF9/ NRas AML cells, either 200 MLL-AF9+/NRas $+\mathrm{GFP}^{+}$or $\mathrm{GFP}^{-}$spleen cells isolated from leukemic mice were plated in methylcellulose media. For serial plating, 2000 cells derived from primary colonies were re-plated. ImageJ software was used to determine colony size.

Cell isolation and FACS analysis. Cells were suspended in Hanks' balanced salt solution (HBSS) (Gibco, Life Technologies) containing 5\% (vol/vol) fetal bovine serum and 2 mM EDTA and prepared for FACS analysis and sorting as previously described ${ }^{67}$. The following antibodies were used to define lineage-positive cells: 145-2C11 (CD3ع), GK1.5 (CD4), 53-6.7 (CD8), RB6-8C5 (Ly-6G/Gr1), M1/70 (CD11b/Mac-1), TER119 (Ly-76/TER119), 6B2 (CD45R/B220), and MB19-1 (CD19). Red blood cells were lysed using RBC Lysis Buffer (eBioscience) before staining for lineage markers. The following additional antibodies were used to define HSC populations: 2B8 (CD117/c-kit), D7 (Ly-6A/E/Sca-1), TC15-12F12.2 (CD150), and A2F10 (CD135/Flt3). All antibodies were purchased from BD Pharmingen, eBioscience or BioLegend. The high throughput surface antibody screen was performed per manufacturer's instruction (BD Lyoplate). In brief, near terminal Msi2-reporter bcCML mice were sacrificed and the spleen was dissociated to produce a single-cell suspension. 500,000 cells were added to each well of a 96 well U-bottom plate. Cells were subsequently incubated with primary, biotinconjugated secondary, and streptavidin-conjugated fluorescent tertiary antibodies while washing twice between antibody steps. Following tertiary antibody incubation and wash, propidium iodide was added and cells were analyzed using a BD FACSCANTO II equipped with a high throughput sampler. All other experiment analysis and cell sorting were carried out on BD LSRFortessa, FACSCanto and FACSAria II and III machines (all from Becton Dickinson) and data were analyzed 
with FlowJo software (Tree Star Inc.). Immunofluorescence images were quantified using ImageJ v.1.52a.

Immunofluorescence staining. Cells were either allowed to settle briefly on polyL-lysine coated chamber slides (VWR) at $37^{\circ} \mathrm{C}$ or cytospun, fixed with $4 \%$ paraformaldehyde (USB Corporation), permeabilized with 1X Dako wash buffer (Dako) and blocked with 10-20\% normal goat serum (Invitrogen) or donkey serum (Abcam) in 1X Dako wash buffer. Primary antibody incubation was overnight at $4{ }^{\circ} \mathrm{C}$. The following primary antibodies were used: rabbit anti-Msi2 1:200 (Abcam), chicken anti-GFP 1:200 (Abcam) and mouse anti-Sdc1 1:100 (Abcam). Alexa fluor-conjugated secondary antibody incubation was performed for $1 \mathrm{~h}$ at room temperature. DAPI (4-6-diamindino-2-phenylindole; Molecular Probes) was used to detect DNA. Images were obtained with a Confocal Leica TCS SP5 II (Leica Microsystems).

Retroviral constructs and production. MIG-BCR-ABL was provided by Warren Pear and Ann Marie Pendergast and was cloned into the MSCV-IRES-NGFR (or MSCV-IRES-YFP) retroviral vector. MSCV-NUP98-HOXA9-IRES-YFP was provided by Gary Gilliland and was sub-cloned into the MSCV-IRES- huCD2 vector (or MSCV-IRES-NGFR) retroviral vector. MSCV-MLL-AF9-IRES-GFP was provided by Scott Armstrong and was sub-cloned into the MSCV-IRES-NGFR retroviral vector. NRASG12V cDNA was a gift from Christopher Counter and was cloned into MSCV-IRES-YFP retroviral vector. Wild-type Sdc1 and Sdc1 HS null constructs were subcloned into MSCV-IRES-GFP retroviral vector. Virus was produced in $293 \mathrm{~T}$ cells transfected using X-tremeGENE HP (Roche) with viral constructs along with VSV-G and gag-pol. Viral supernatants were collected for 3-6 days followed by the ultracentrifugal concentration at $20,000 \times g$ for $2 \mathrm{~h}$.

qRT-PCR analysis. RNA was isolated using RNeasy micro kit (Qiagen) and RNA was converted to cDNA using Superscript III reverse transcriptase (Life Technologies). Quantitative real-time PCRs were performed using an iCycler (BioRad) by mixing cDNAs, iQ SYBRGreen Supermix (BioRad) and gene-specific primers. Gene expression was normalized to the levels of Beta-2 microglobulin (B2M) except for Supplementary Fig. 1c that was normalized to beta-actin (Actb). All primers were designed using NCBI's Primer-BLAST (https://www.ncbi.nlm.nih. gov/tools/primer-blast/). Primer sequences are listed in Supplementary Data 2.

Live animal imaging. Live mice were imaged as described previously. In brief, mice were anaesthetized by intraperitoneal injection of ketamine and xylazine (100 and $20 \mathrm{mg} / \mathrm{kg}$ respectively). Once unresponsive to pedal reflex, hair was removed using Nair Hair Remover lotion (Church \& Dwight Co.) and the scalp was removed to expose the calvarium. Mice were then secured into a mouse/neonatal rat stereotactic holder (Stoelting Co.). The topmost layer of bone was removed with a Dremel 3000 variable speed rotary tool equipped with a 1/16" engraving cutter bit and a $1 \mathrm{~cm}$ rubber O-ring was attached to the exposed calvarium (Fine Science Tools Inc.). Images were acquired by Leica LAS AF 2.7.3.9723 software with an upright Leica SP5 2 confocal system using a Leica DM 6000 CFS microscope. Images were acquired using an HCX APO L20 objective with a 1.0 numerical aperture. Resultant videos were adjusted using Adobe Photoshop CS6 version 13.0.

Transwell chemotaxis assay. Directed cell migration towards SDF1 was analyzed in vitro. Primary murine bcCML was sorted and the Lineage ${ }^{-}$fraction was transduced with either control shRNA or shSdcl. After $48 \mathrm{~h}$, transduced cells were sorted again for the presence of the shRNA viral construct. Cells were washed, resuspended in IMDM $+0.25 \% \mathrm{BSA}$, and plated in the apical chamber of $6.5 \mathrm{~mm}$ transwell filters (Costar, pore size $5 \mu \mathrm{m}$ ) that had been coated with fibronectin (Sigma Aldrich) or MAdCAM-1 (R\&D Systems) overnight. Six hundred microliters of IMDM $+0.25 \%$ BSA $+100 \mathrm{ng} / \mathrm{mL}$ SDF-1 (R\&D Systems) was added to the basal chamber. Cells were allowed to migrate for $4 \mathrm{~h}$ at $37^{\circ} \mathrm{C}$ in a $5 \% \mathrm{CO}_{2}$ incubator. After incubation, migrated cells were collected from the lower chamber and counted using Trypan Blue. For migration across HUVEC monolayers, fibronectincoated transwells were seeded with HUVECs, and allowed to establish a monolayer. The night before running the experiment, HUVECs were activated with $20 \mathrm{ng} / \mathrm{mL}$ TNF- $\alpha$ (BD Biosciences). The experiment then proceeded as above.

Polyacrylamide gel chemotaxis assay. Chemically activated polyacrylamide gels on $18 \mathrm{~mm}$ coverslips ( $8 \mathrm{kPa}$, Matrigen) were coated with $1 \mu \mathrm{g} / \mathrm{mL}$ fibronectin (Sigma Aldrich) before seeding HUVECs. HUVEC monolayer establishment, and activation were as described above. The day of the assay, a chemoattractant gradient was established by incubation of gels with $1 \mu \mathrm{g} / \mathrm{mL}$ SDF- 1 for $>2 \mathrm{~h}$ at $37^{\circ} \mathrm{C}$ in a $5 \% \mathrm{CO}_{2}$ incubator. HUVEC monolayers were stained with CellMask Orange Plasma membrane stain (Invitrogen) before sorted shSdcl or shCTRL bcCML cells were washed, resuspended in IMDM $+0.25 \% \mathrm{BSA}$, and added to the gels. Gels/ cells were maintained at $37^{\circ} \mathrm{C}$ and $5 \% \mathrm{CO}_{2}$ while imaging. Images were obtained using a Zeiss LSM-700 confocal microscope.
WT vs. Sdc1 ${ }^{-1}$ - bcCML RNAseq. Lin $^{-}$leukemic cells were sorted from mice transplanted with BCR-ABL/NUP98-HOXA9 transduced KLS cells. KLS cells were originally sorted from either WT or $\mathrm{Sdc1}^{-/-}$mice to generate WT or knockout leukemia, respectively. Total RNA was isolated using the RNeasy Micro Plus kit (QIAGEN). RNA libraries were generated from $150 \mathrm{ng}$ of RNA using Illumina's TruSeq Stranded mRNA Sample Prep Kit (Illumina). Libraries were pooled and single-end sequenced $(1 \times 75)$ on the Illumina NextSeq 500 using the High output V2 kit (Illumina).

RNAseq and cell state analysis. Sdc1 KO and WT RNAseq fastq files were processed into transcript-level summaries using kallisto $^{68}$. Transcript-level summaries were processed into gene-level summaries and differential gene expression was performed using sleuth with the Wald test ${ }^{69}$. GSEA was performed as previously described ${ }^{70}$. ClueGO was used for gene enrichment analysis of downregulated genes (bias estimator $>0.5$ and FDR $<0.05$ ) identified between WT and Sdc1 KO mouse cells. PID and Halllmark gene sets were used with medium network specificity, a $p$-value cutoff of $<0.05$ and a kappa score of 0.4 . All other statistical parameters remained on default settings. CluePedia was used to identify genes found within enriched gene sets. All network analyses ran and visualized in Cytoscape 3.7 (Cytoscape, ClueGO/CluePedia).

Soluble ligand binding assay. $5 \times 10^{6}$ cells were washed and resuspended in HBSS containing $0.1 \% \mathrm{BSA}$ and $1 \mathrm{mM} \mathrm{Ca}{ }^{2+} / \mathrm{Mg}^{2+}$, prior to incubation with integrin ligands $(10 \mu \mathrm{g} / \mathrm{ml}$ mouse MAdCAM-1 or $5 \mu \mathrm{g} / \mathrm{ml}$ VCAM- 1$)$ for $30 \mathrm{~min}$ at $37^{\circ} \mathrm{C}$ with or without $200 \mathrm{nM}$ PMA or $1 \mathrm{mM} \mathrm{Mn}^{2+}$. Cells were then incubated with AlexFluor647-conjugated anti-human IgG (1:200) for $30 \mathrm{~min}$ at $4{ }^{\circ} \mathrm{C}$. Then cells were washed twice before flow cytometry analysis using an Accuri C6 Plus or FACSCalibur (BD Biosciences). Data were analyzed using FlowJo software:

$$
\text { Relative activation index }=\frac{M F I-M_{\mathrm{EDTA}}}{\mathrm{MFI}_{\mathrm{Mn}}-\mathrm{MFI}_{\mathrm{EDTA}}}
$$

Statistics and reproducibility. Statistical analyses were carried out using GraphPad Prism software version 6.0f-h (GraphPad software Inc.). All data are shown as mean \pm SEM. Two-tailed unpaired Student's $t$-tests were used to determine statistical significance. No statistical method was used to predetermine sample size and no data were excluded from the analysis. All experiments were reproducible.

Reporting summary. Further information on research design is available in the Nature Research Reporting Summary linked to this article.

\section{Data availability}

Source data are provided with this paper as a separate Source Data file. Antibody information is provided in Supplementary Data 1. Primer information is provided in Supplementary Data 2. Examples of flow cytometry gating strategies are provided as Supplementary Fig. 6. RNAseq data sets have been deposited into the NCBI GEO database under the accession code GSE159148 and are available through the following link. All other data supporting the findings of this study are available within the article and its supplementary information files and from the corresponding author upon reasonable request. Source data are provided with this paper.

Received: 13 March 2020; Accepted: 29 October 2020; Published online: 26 November 2020

\section{References}

1. O'Hare, T., Corbin, A. S. \& Druker, B. J. Targeted CML therapy: controlling drug resistance, seeking cure. Curr. Opin. Genet. Dev. 16, $92-99$ (2006).

2. Oravecz-Wilson, K. I. et al. Persistence of leukemia-initiating cells in a conditional knockin model of an imatinib-responsive myeloproliferative disorder. Cancer Cell 16, 137-148 (2009).

3. $\mathrm{Li}, \mathrm{X}$. et al. Intrinsic resistance of tumorigenic breast cancer cells to chemotherapy. J. Natl Cancer Inst. 100, 672-679 (2008).

4. Bao, S. et al. Glioma stem cells promote radioresistance by preferential activation of the DNA damage response. Nature 444, 756-760 (2006).

5. Diehn, M. et al. Association of reactive oxygen species levels and radioresistance in cancer stem cells. Nature 458, 780-783 (2009).

6. Holyoake, T., Jiang, X., Eaves, C. \& Eaves, A. Isolation of a highly quiescent subpopulation of primitive leukemic cells in chronic myeloid leukemia. Blood 94, 2056-2064 (1999).

7. Chu, S. et al. Detection of BCR-ABL kinase mutations in CD34+ cells from chronic myelogenous leukemia patients in complete cytogenetic remission on imatinib mesylate treatment. Blood 105, 2093-2098 (2005). 
8. Jiang, X., Saw, K. M., Eaves, A. \& Eaves, C. Instability of BCR-ABL gene in primary and cultured chronic myeloid leukemia stem cells. J. Natl Cancer Inst. 99, 680-693 (2007).

9. Konig, H., Holyoake, T. L. \& Bhatia, R. Effective and selective inhibition of chronic myeloid leukemia primitive hematopoietic progenitors by the dual Src/Abl kinase inhibitor SKI-606. Blood 111, 2329-2338 (2008)

10. Corbin, A. S. et al. Human chronic myeloid leukemia stem cells are insensitive to imatinib despite inhibition of BCR-ABL activity. J. Clin. Invest 121, 396-409 (2011).

11. Bonnet, D. \& Dick, J. E. Human acute myeloid leukemia is organized as a hierarchy that originates from a primitive hematopoietic cell. Nat. Med. 3, 730-737 (1997).

12. Lapidot, T. et al. A cell initiating human acute myeloid leukaemia after transplantation into SCID mice. Nature 367, 645-648 (1994).

13. Costello, R. T. et al. Human acute myeloid leukemia CD34+/CD38progenitor cells have decreased sensitivity to chemotherapy and Fas-induced apoptosis, reduced immunogenicity, and impaired dendritic cell transformation capacities. Cancer Res. 60, 4403-4411 (2000).

14. Al-Hajj, M., Wicha, M. S., Benito-Hernandez, A., Morrison, S. J. \& Clarke, M. F. Prospective identification of tumorigenic breast cancer cells. Proc. Natl Acad. Sci. USA 100, 3983-3988 (2003).

15. Ricci-Vitiani, L. et al. Identification and expansion of human colon-cancerinitiating cells. Nature 445, 111-115 (2007).

16. Jordan, C. T., Guzman, M. L. \& Noble, M. Cancer stem cells. N. Engl. J. Med. 355, 1253-1261 (2006).

17. Todaro, M. et al. CD44v6 is a marker of constitutive and reprogrammed cancer stem cells driving colon cancer metastasis. Cell Stem Cell 14, 342-356 (2014).

18. Fox, R. G., Park, F. D., Koechlein, C. S., Kritzik, M. \& Reya, T. Musashi signaling in stem cells and cancer. Annu. Rev. Cell Dev. Biol. 31, 249-267 (2015).

19. Koechlein, C. S. et al. High-resolution imaging and computational analysis of haematopoietic cell dynamics in vivo. Nat. Commun. 7, 12169 (2016).

20. Fox, R. G. et al. Image-based detection and targeting of therapy resistance in pancreatic adenocarcinoma. Nature 534, 407-411 (2016).

21. Dash, A. B. et al. A murine model of CML blast crisis induced by cooperation between BCR/ABL and NUP98/HOXA9. Proc. Natl Acad. Sci. USA 99, 7622-7627 (2002).

22. Neering, S. J. et al. Leukemia stem cells in a genetically defined murine model of blast-crisis CML. Blood 110, 2578-2585 (2007).

23. Eppert, K. et al. Stem cell gene expression programs influence clinical outcome in human leukemia. Nat. Med. 17, 1086-1093 (2011)

24. Somervaille, T. C. \& Cleary, M. L. Identification and characterization of leukemia stem cells in murine MLL-AF9 acute myeloid leukemia. Cancer Cell 10, 257-268 (2006)

25. Kantarjian, H. et al. Hematologic and cytogenetic responses to imatinib mesylate in chronic myelogenous leukemia. N. Engl. J. Med. 346, 645-652 (2002).

26. Shah, N. P. et al. Multiple BCR-ABL kinase domain mutations confer polyclonal resistance to the tyrosine kinase inhibitor imatinib (STI571) in chronic phase and blast crisis chronic myeloid leukemia. Cancer Cell 2, 117-125 (2002).

27. Nair, P. R. et al. Filomicelles from aromatic diblock copolymers increase paclitaxel-induced tumor cell death and aneuploidy compared with aliphatic copolymers. Nanomedicine11, 1551-1569 (2016).

28. Drexler, H. G., Zaborski, M. \& Quentmeier, H. Interferon-gamma induced proliferation of human myeloid leukaemia cell lines. Br. J. Haematol. 98, 699-710 (1997).

29. Mumprecht, S., Schurch, C., Schwaller, J., Solenthaler, M. \& Ochsenbein, A. F. Programmed death 1 signaling on chronic myeloid leukemia-specific $\mathrm{T}$ cells results in T-cell exhaustion and disease progression. Blood 114, 1528-1536 (2009).

30. Stepp, M. A., Pal-Ghosh, S., Tadvalkar, G. \& Pajoohesh-Ganji, A. Syndecan-1 and ITs Expanding List of Contacts. Adv. Wound Care 4, 235-249 (2015).

31. Xu, D. \& Esko, J. D. Demystifying heparan sulfate-protein interactions. Annu Rev. Biochem 83, 129-157 (2014).

32. Seftalioglu, A. \& Karakus, S. Syndecan-1/CD138 expression in normal myeloid, acute lymphoblastic and myeloblastic leukemia cells. Acta Histochem. 105, 213-221 (2003).

33. Stepp, M. A. et al. Defects in keratinocyte activation during wound healing in the syndecan-1-deficient mouse. J. Cell Sci. 115, 4517-4531 (2002).

34. Beauvais, D. M. \& Rapraeger, A. C. Syndecan-1 couples the insulin-like growth factor-1 receptor to inside-out integrin activation. J. Cell Sci. 123, 3796-3807 (2010).

35. Beauvais, D. M., Ell, B. J., McWhorter, A. R. \& Rapraeger, A. C. Syndecan-1 regulates alphavbeta 3 and alphavbeta5 integrin activation during angiogenesis and is blocked by synstatin, a novel peptide inhibitor. J. Exp. Med. 206 691-705 (2009).

36. Chen, B., Kumar, G., Co, C. C. \& Ho, C. C. Geometric control of cell migration. Sci. Rep. 3, 2827 (2013).
37. Bajaj, J. et al. An in vivo genome-wide CRISPR screen identifies the RNAbinidng protein Staufen2 as a key regulator of myeloid leukemia. Nat. Cancer 1, 410-422 (2020)

38. Chen, J. et al. A restricted cell population propagates glioblastoma growth after chemotherapy. Nature 488, 522-526 (2012).

39. Boumahdi, S. et al. SOX2 controls tumour initiation and cancer stem-cell functions in squamous-cell carcinoma. Nature 511, 246-250 (2014).

40. Yassin, M. et al. A novel method for detecting the cellular stemness state in normal and leukemic human hematopoietic cells can predict disease outcome and drug sensitivity. Leukemia 33, 2061-2077 (2019).

41. Kudinov, A. E., Karanicolas, J., Golemis, E. A. \& Boumber, Y. Musashi RNAbinding proteins as cancer drivers and novel therapeutic targets. Clin. Cancer Res. 23, 2143-2153 (2017).

42. Gotte, M., Kersting, C., Radke, I., Kiesel, L. \& Wulfing, P. An expression signature of syndecan-1 (CD138), E-cadherin and c-met is associated with factors of angiogenesis and lymphangiogenesis in ductal breast carcinoma in situ. Breast Cancer Res. 9, R8 (2007).

43. Ibrahim, S. A. et al. Syndecan-1 (CD138) modulates triple-negative breast cancer stem cell properties via regulation of LRP-6 and IL-6-mediated STAT3 signaling. PLoS ONE 8, e85737 (2013)

44. Jung, O. et al. Heparanase-induced shedding of syndecan-1/CD138 in myeloma and endothelial cells activates VEGFR2 and an invasive phenotype: prevention by novel synstatins. Oncogenesis 5, e202 (2016).

45. Schonfeld, K. et al. Indatuximab ravtansine (BT062) combination treatment in multiple myeloma: pre-clinical studies. J. Hematol. Oncol. 10, 13 (2017).

46. Lin, P., Owens, R., Tricot, G. \& Wilson, C. S. Flow cytometric immunophenotypic analysis of 306 cases of multiple myeloma. Am. J. Clin. Pathol. 121, 482-488 (2004)

47. Spiegel, A. et al. Unique SDF-1-induced activation of human precursor-B ALL cells as a result of altered CXCR4 expression and signaling. Blood 103 2900-2907 (2004)

48. Shen, W., Bendall, L. J., Gottlieb, D. J. \& Bradstock, K. F. The chemokine receptor CXCR4 enhances integrin-mediated in vitro adhesion and facilitates engraftment of leukemic precursor-B cells in the bone marrow. Exp. Hematol. 29, 1439-1447 (2001).

49. Jin, L., Hope, K. J., Zhai, Q., Smadja-Joffe, F. \& Dick, J. E. Targeting of CD44 eradicates human acute myeloid leukemic stem cells. Nat. Med. 12, 1167-1174 (2006).

50. Krause, D. S., Lazarides, K., von Andrian, U. H. \& Van Etten, R. A. Requirement for CD44 in homing and engraftment of BCR-ABL-expressing leukemic stem cells. Nat. Med. 12, 1175-1180 (2006).

51. Alexopoulou, A. N., Multhaupt, H. A. \& Couchman, J. R. Syndecans in wound healing, inflammation and vascular biology. Int J. Biochem Cell Biol. 39, 505-528 (2007).

52. Bernfield, M. et al. Functions of cell surface heparan sulfate proteoglycans. Annu. Rev. Biochem. 68, 729-777 (1999).

53. Woods, A. Syndecans: transmembrane modulators of adhesion and matrix assembly. J. Clin. Invest. 107, 935-941 (2001).

54. Beauvais, D. M., Burbach, B. J. \& Rapraeger, A. C. The syndecan-1 ectodomain regulates alphavbeta3 integrin activity in human mammary carcinoma cells. J. Cell Biol. 167, 171-181 (2004).

55. Matsumoto, Y. et al. Trimeric Tn antigen on syndecan 1 produced by ppGalNAc-T13 enhances cancer metastasis via a complex formation with integrin alpha5betal and matrix metalloproteinase 9. J. Biol. Chem. $\mathbf{2 8 8}$ 24264-24276 (2013)

56. Corces-Zimmerman, M. R., Hong, W. J., Weissman, I. L., Medeiros, B. C. \& Majeti, R. Preleukemic mutations in human acute myeloid leukemia affect epigenetic regulators and persist in remission. Proc. Natl Acad. Sci. USA 111, 2548-2553 (2014)

57. Jacamo, R. et al. Reciprocal leukemia-stroma VCAM-1/VLA-4-dependent activation of NF-kappaB mediates chemoresistance. Blood 123, 2691-2702 (2014).

58. Matsunaga, T. et al. Interaction between leukemic-cell VLA-4 and stromal fibronectin is a decisive factor for minimal residual disease of acute myelogenous leukemia. Nat. Med. 9, 1158-1165 (2003).

59. Bajaj, J. et al. CD98-mediated adhesive signaling enables the establishment and propagation of acute myelogenous leukemia. Cancer Cell 30, 792-805 (2016).

60. Imai, T. et al. The neural RNA-binding protein Musashil translationally regulates mammalian numb gene expression by interacting with its mRNA. Mol. Cell Biol. 21, 3888-3900 (2001).

61. Zearfoss, N. R. et al. A conserved three-nucleotide core motif defines Musashi RNA binding specificity. J. Biol. Chem. 289, 35530-35541 (2014).

62. Vihinen, T., Maatta, A., Jaakkola, P., Auvinen, P. \& Jalkanen, M. Functional characterization of mouse syndecan-1 promoter. J. Biol. Chem. 271, 12532-12541 (1996).

63. Wang, H., Jin, H., Beauvais, D. M. \& Rapraeger, A. C. Cytoplasmic domain interactions of syndecan- 1 and syndecan- 4 with alpha6beta 4 integrin mediate human epidermal growth factor receptor (HER1 and HER2)-dependent motility and survival. J. Biol. Chem. 289, 30318-30332 (2014). 
64. Hosen, N. et al. The activated conformation of integrin beta7 is a nove multiple myeloma-specific target for CAR T cell therapy. Nat. Med. 23 , 1436-1443 (2017).

65. Kwon, H. Y. et al. Tetraspanin 3 is required for the development and propagation of acute myelogenous leukemia. Cell Stem Cell 17, 152-164 (2015).

66. Zimdahl, B. et al. Lis1 regulates asymmetric division in hematopoietic stem cells and in leukemia. Nat. Genet. 46, 245-252 (2014).

67. Domen, J., Cheshier, S. H. \& Weissman, I. L. The role of apoptosis in the regulation of hematopoietic stem cells: overexpression of Bcl-2 increases both their number and repopulation potential. J. Exp. Med. 191, 253-264 (2000)

68. Bray, N. L. et al. Near-optimal probabilistic RNA-seq quantification. Nature Biotechnology 34, 525-527 (2016).

69. Pimentel, H. et al. Differential analysis of RNA-seq incorporating quantification uncertainty. Nature Methods 14, 687-690 (2017).

70. Lytle, N. K. et al. A Multiscale Map of the Stem Cell State in Pancreatic Adenocarcinoma. Cell 177, 572-586.e22 (2019).

\section{Acknowledgements}

We would like to thank Becton Dickinson for making mAb screening available, Warren Pear and Ann Marie Pendergast for providing MIG-BCR-ABL, Gary Gilliland for providing MSCV-NUP98-HOXA9-IRES-YFP, Scott Armstrong for providing MSCVMLL-AF9-IRES-GFP, and Christopher Counter for providing NRAS G12V cDNA. K.S. received support from NIH grants T32CA009523 and T32HL086344. J.B. was supported by a postdoctoral fellowship from the National Cancer Center and an ASH Scholar Award. M.H. received support from NIH grant T32HL086344. N.K.L. received support from T32 GM007752 and National Research Service Award F31 CA206416. C.S.K. received support from T32 GM007752. B.Z. received support from T32 GM007184 (Duke University). T.I. was the recipient of a California Institute for Regenerative Medicine interdisciplinary stem cell training program fellowship. This work was supported by NIH grant HL151433 to M.H.G., GM33063 to J.D.E. and NIH grants DP1 CA174422 and R35 CA197699 to T.R. This work is dedicated to Joi Weeks, who lost her life to cancer, and inspires us to continue the fight.

\section{Author contributions}

K.S. designed and performed experiments and helped write the paper; J.B. provided experimental advice and support; T.I., B.Z., A.A., C.K., N.L., H.Y.K., F.A-E-K., H.S., A.B., and J.W. provided experimental support; M.H., performed bioinformatics analysis related to RNAseq and network analysis; M.K. helped write the paper; J.K., M.H.G., and J.D.E. provided experimental advice; P.W.P. provided Sdcl antibody and provided experimental advice; T.R. conceived the project, planned and guided the research and wrote the paper.

\section{Competing interests}

The authors declare no competing interests.

\section{Additional information}

Supplementary information is available for this paper at https://doi.org/10.1038/s41467020-19782-x.

Correspondence and requests for materials should be addressed to T.R.

Peer review information Nature Communications thanks the anonymous reviewer(s) for their contribution to the peer review of this work.

Reprints and permission information is available at http://www.nature.com/reprints

Publisher's note Springer Nature remains neutral with regard to jurisdictional claims in published maps and institutional affiliations.

\section{(c) (i)}

Open Access This article is licensed under a Creative Commons Attribution 4.0 International License, which permits use, sharing, adaptation, distribution and reproduction in any medium or format, as long as you give appropriate credit to the original author(s) and the source, provide a link to the Creative Commons license, and indicate if changes were made. The images or other third party material in this article are included in the article's Creative Commons license, unless indicated otherwise in a credit line to the material. If material is not included in the article's Creative Commons license and your intended use is not permitted by statutory regulation or exceeds the permitted use, you will need to obtain permission directly from the copyright holder. To view a copy of this license, visit http://creativecommons.org/ licenses/by/4.0\%.

(c) The Author(s) 2020 